\title{
Association between the baseline tumor size and outcomes of patients with non-small cell lung cancer treated with first-line immune checkpoint inhibitor monotherapy or in combination with chemotherapy
}

\author{
Yuji Uehara^, Taiki Hakozaki^, Rui Kitadai, Kosuke Narita, Kageaki Watanabe, Kana Hashimoto, \\ Shoko Kawai, Makiko Yomota, Yukio Hosomi \\ Department of Thoracic Oncology and Respiratory Medicine, Tokyo Metropolitan Cancer and Infectious Diseases Center, Komagome Hospital, \\ Tokyo, Japan \\ Contributions: (I) Conception and design: T Hakozaki, Y Uehara; (II) Administrative support: T Hakozaki; (III) Provision of study materials or \\ patients: T Hakozaki; (IV) Collection and assembly of data: Y Uehara, T Hakozaki, R Kitadai; (V) Data analysis and interpretation: Y Uehara, T \\ Hakozaki; (VI) Manuscript writing: All authors; (VII) Final approval of manuscript: All authors. \\ Correspondence to: Taiki Hakozaki, MD. Department of Thoracic Oncology and Respiratory Medicine, Tokyo Metropolitan Cancer and Infectious \\ Diseases Center, Komagome Hospital, 3-18-22 Honkomagome, Bunkyo, Tokyo, Japan. Email: t-hakozaki@cick.jp.
}

\begin{abstract}
Background: The baseline tumor size (BTS) is a prognostic factor for patients with non-small cell lung cancer (NSCLC) who received immune checkpoint inhibitor monotherapy (ICI-mono). However, this relationship is not yet known in patients treated with ICI in combination with chemotherapy (ICI-chemo).

Methods: This single-center retrospective study evaluated 159 patients with advanced NSCLC who received first-line ICI-mono or ICI-chemo from January 2016 to April 2021. Their BTS values were estimated using the maximum BTS (max BTS) (maximum target lesions' longest diameter) and total BTS (sum of target lesions' longest diameters) in a radiological assessment according to the Response Evaluation Criteria for Solid Tumors.

Results: Based on a multivariable analysis, the large max BTS group had worse progression-free survival (PFS) in patients treated with ICI-mono $(\mathrm{P}=0.009)$, but it was not associated with worse PFS in patients treated with ICI-chemo $(\mathrm{P}=0.132)$. The group treated with ICI-mono had worse PFS compared to the group treated with ICI-chemo in patients with $\max$ BTS $\geq 50 \mathrm{~mm}(\mathrm{P}=0.004)$, and the group treated with ICI-mono was not associated with worse PFS compared to the group treated with ICI-chemo in patients with max BTS $<50 \mathrm{~mm}(\mathrm{P}=0.107)$.

Conclusions: While a large max BTS was identified as a prognostic factor for worse PFS in patients treated with ICI-mono, it was not identified as such in patients treated with ICI-chemo. The max BTS may have different predicting efficacy for patients with NSCLC treated with ICI-mono and ICI-chemo.
\end{abstract}

Keywords: Baseline tumor burden; immunotherapy; pembrolizumab; progression; survival

Submitted Oct 04, 2021. Accepted for publication Jan 23, 2022.

doi: $10.21037 /$ tlcr-21-815

View this article at: https://dx.doi.org/10.21037/tlcr-21-815

^ ORCID: Yuji Uehara, 0000-0001-8047-8730; Taiki Hakozaki, 0000-0002-9980-4417. 


\section{Introduction}

In recent years, our choice of standard therapeutic options for patients with metastatic non-small cell lung cancer (NSCLC) has been expanded by immune checkpoint inhibitors (ICIs) either as a monotherapy or in combination with chemotherapy. Some patients treated with ICI have remarkably long-lasting responses and survival. However, unique toxicities and immune-related adverse events occur at any point during or after the treatment. Therefore, it is important to identify better clinical biomarkers that can predict the response to ICI. Few clinical biomarkers have been validated to predict the response of NSCLC to ICI other than through programmed death ligand-1 (PD-L1) expression (1-3).

In a radiological assessment, the baseline tumor size (BTS) has emerged as an independent prognostic factor of survival of patients treated with pembrolizumab monotherapy (4). Baseline imaging findings are crucial to determine the staging according to tumor, node, and metastasis (TNM) classifications in daily practice. Imaging profiles, such as tumor size and metastatic lesions, are also commonly considered by clinicians for decision-making. Previous studies have reported that BTS and the number of organs involved before treatment were prognostic factors for the efficacy of ICI monotherapy (ICI-mono) in patients with melanoma, head and neck squamous cell carcinoma, and NSCLC (5-10). Particularly, the maximum BTS (max BTS) was reported to be a feasible clinical biomarker for the efficacy of ICI in everyday practice (11). However, the association between BTS and clinical outcomes is still unknown in patients with ICI in combination with chemotherapy (ICI-chemo). Moreover, the benefit of adding chemotherapy to ICI remains unclear in PD-L1 $\geq 50 \%$, although it has been developed as a strategy to overcome primary resistance $(3,12)$. Several meta-analyses comparing ICI-mono $v s$. ICI-combo presented paradoxical results, which might be attributed to inherent limitations, such as the risk of systematic bias and confounding factors (13-16). Despite its clinical importance, there is a lack of research investigating BTS as a predictive biomarker to select between ICI-mono or ICI-chemo.

The purpose of this retrospective study was to investigate BTS as a prognostic and predictive factor for the efficacy of first-line ICI-mono and ICI-chemo in patients with NSCLC. To assess the association between the max BTS and outcomes, a radiological assessment of the max BTS (maximum target lesions' longest diameter), total BTS (sum of target lesions' longest diameters), and number of organs involved was performed in accordance with previous studies $(7,11)$. The associations between the max BTS and clinical outcomes of Japanese patients with advanced NSCLC were investigated.

We present the following article in accordance with the STROBE reporting checklist (available at https://tlcr. amegroups.com/article/view/10.21037/tlcr-21-815/rc).

\section{Methods}

\section{Patients}

From January 2016 to April 2021, 191 patients with advanced NSCLC underwent ICI-mono or ICI-chemo as a first-line treatment at the Department of Thoracic Oncology and Respiratory Medicine at the Tokyo Metropolitan Cancer and Infectious Diseases Center of Komagome Hospital (Tokyo, Japan). The patients' medical records were retrospectively reviewed to collect data on age, sex, histological subtypes, smoking history, and Eastern Cooperative Oncology Group performance status (ECOGPS) at the first dose of programmed death-1 (PD-1)/PD-L1 inhibitor treatment, lactate dehydrogenase (LDH) levels, neutrophil-to-lymphocyte ratio (NLR), and lung immune prognostic index (LIPI), which is calculated from LDH and NLR values (2). All procedures performed in this study involving human participants were in accordance with the Declaration of Helsinki (as revised in 2013). The study was approved by the institutional review boards of Tokyo Metropolitan Cancer and Infectious Diseases Center of Komagome Hospital (No. 2725). Individual consent for this retrospective analysis was waived.

\section{Evaluation of BTS}

BTS was evaluated using computed tomography or magnetic resonance imaging and the Response Evaluation Criteria for Solid Tumors version 1.1. Up to a maximum of two measurable lesions per organ and five measurable lesions in total were identified. The target lesions required the largest diameter to be $\geq 10 \mathrm{~mm}$ or the short axis to be $\geq 15 \mathrm{~mm}$ to be considered a lymph node lesion. BTS was estimated using the max BTS (maximum target lesions' longest diameter) and total BTS (sum of target lesions' longest diameters). The number of organs involved was defined using both target and nontarget lesions. Positive thoracic lymph nodes $(\mathrm{N} 1-\mathrm{N} 3)$, including those in the 
supraclavicular fossae, were counted as a single organ.

\section{Statistical analysis}

Descriptive statistics were used for the patients' and tumor characteristics. Data are presented as relative frequencies (percentage) or median and interquartile range (IQR) for continuous variables. Fisher's exact tests for categorical data and Mann-Whitney $U$ test for continuous variables were used to assess the intergroup differences at baseline and the clinical response to ICI treatment.

A cutoff point was identified to investigate the independent prognostic role of BTS using the minimum $\mathrm{P}$ value method, which selects a cut-point using the maximum $\chi^{2}$ statistic as the optimal cut-point when the outcomes are binary (17). The relationship between the tumor mass (max BTS, total BTS, and number of organs involved) and progression-free survival (PFS) was tested using the minimum $\mathrm{P}$ value method of the log-rank test.

The clinical benefit rate (CBR) was defined as the proportion of patients experiencing the best overall response of complete response (CR), partial response (PR), or stable disease (SD) for $\geq 6$ months. PFS was calculated from the start of the first-line immunotherapy to the first evidence of disease progression, death from any cause, or the last follow-up (censored). The overall survival (OS) interval was calculated from the start of the firstline immunotherapy to death from any reason or the last follow-up (censored). The end of the follow-up period was on June 7, 2021. The median PFS and OS were compared using the Kaplan-Meier method and log-rank test. The restricted mean survival time (RMST) was conducted to estimate cancer-specific survival differences during a 12- or 18-month period (18). Factors associated with PFS based on the univariate analyses were considered for multivariate models. Multivariate Cox proportional hazard models were used to investigate the independent prognostic role of the max BTS, adjusting for other prognostic factors and confounders.

For all analyses, results are presented as hazard ratios (HRs) with 95\% confidence intervals (CIs), and two-tailed $\mathrm{P}<0.05$ was considered statistically significant. The statistical analyses were performed with a graphical user interface for R (The R Foundation for Statistical Computing, Vienna, Austria).

\section{Results}

\section{Patient characteristics}

Of the 191 potentially eligible patients, we excluded 23 patients with recurrence after chemoradiotherapy because their patients included the previous durvalumab treatment. Additionally, we excluded one patient with EGFR/ALK/ $R O S 1 / B R A F$ alterations and five clinical trial patients who received experimental regimens unapproved by FDA, thus no mutations in $E G F R / A L K / R O S 1 / B R A F$ were identified in our cohort (Figure S1). Therefore, 159 patients were ultimately included in this study. The pretreatment characteristics of the study participants are described in Table 1. In total, 80 patients $(50.3 \%)$ received ICI-mono, and 79 patients $(49.7 \%)$ received ICI-chemo. The median age was 71 (range, $31-89$ ) years, and $80.5 \%$ of the patients were male. Moreover, 128 (80.5\%) and 31 (19.5\%) patients had a performance status of $<2$ and $\geq 2$, respectively; 104 patients $(65.4 \%)$ had adenocarcinoma, 38 patients $(23.9 \%)$ had squamous cell carcinoma, and 17 patients (10.7\%) had not otherwise specified (NOS) carcinoma. Based on the 8th edition of the TNM classification for lung cancer, 32 (20.1\%), 10 (6.3\%), 44 (27.7\%), and 73 (45.9\%) patients presented with recurrent, stage III, stage IVA, and stage IVB disease, respectively. The results for PD-L1 expression were available for $143(89.9 \%)$ patients, with a high PDL1 tumor proportion score (TPS) $(\geq 50 \%)$ identified in $86(54.1 \%)$ patients. Meanwhile, 151 patients $(95.0 \%)$ received pembrolizumab regimens, and eight patients $(5.0 \%)$ received atezolizumab regimens. The median max BTS, total BTS, and number of organs involved for all patients were 54.2 (range, 37.2-72.5) mm, 104.3 (range, 67.7-151.1) mm, and 3.0 (IQR, 2.0-4.0), respectively. The patients' baseline demographic and disease characteristics were generally well-balanced between the ICI-mono and ICI-chemo groups; except more patients with aged $\geq 75$ years, $\mathrm{PS} \geq 2$, recurrence at staging, or PD-L1 TPS $\geq 50 \%$ were in the ICI-mono group $(\mathrm{P}<0.001, \mathrm{P}<0.001$, $\mathrm{P}=0.006$, and $\mathrm{P}<0.001$, respectively). The most common site for the largest target lesions was a primary pulmonary lesion (Table S1).

\section{Cutoff values to investigate the prognostic role of the BTS}

Using the minimum $\mathrm{P}$ value method, the optimal cutoff 
Table 1 Patient baseline characteristics

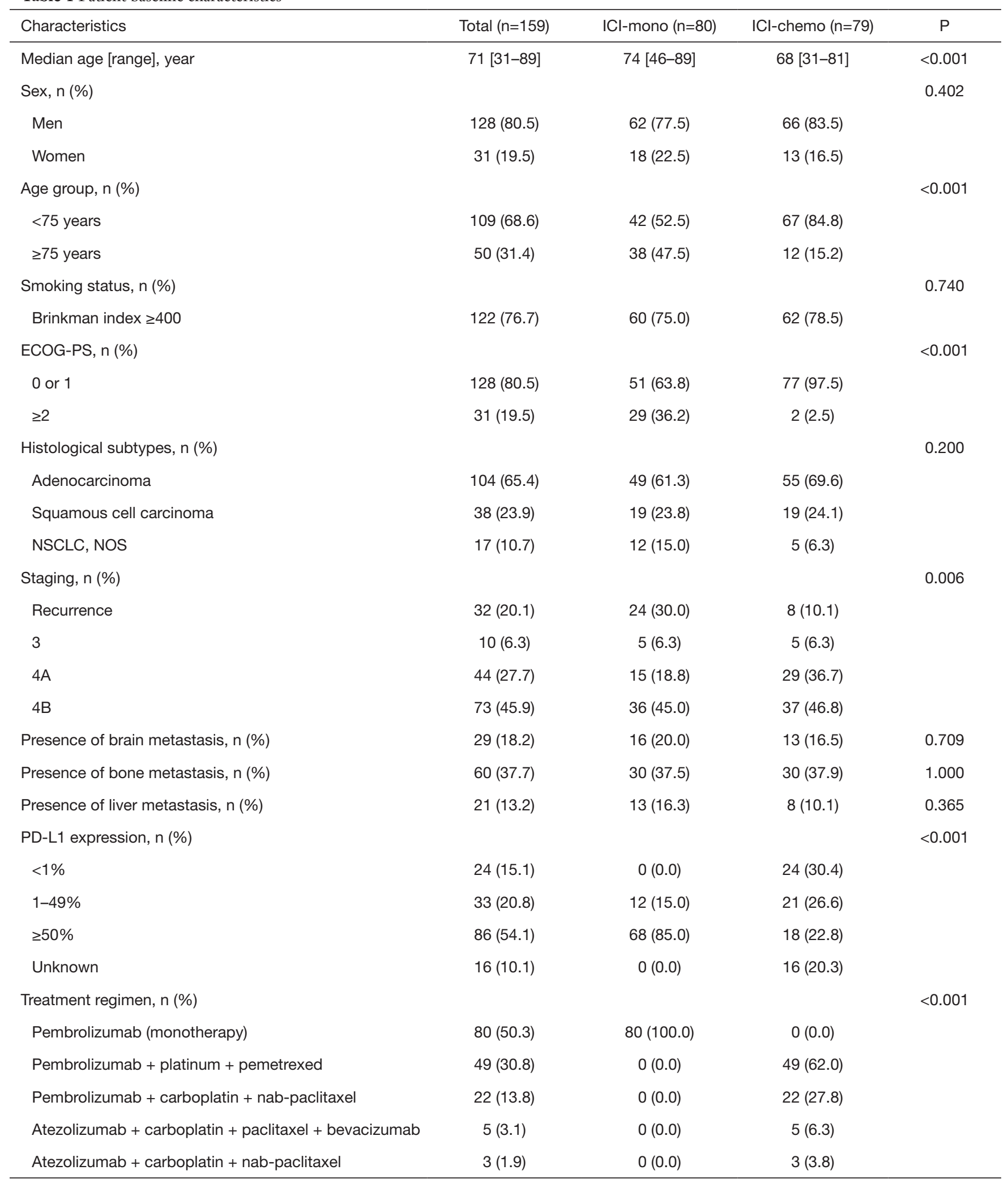

Table 1 (continued) 
Table 1 (continued)

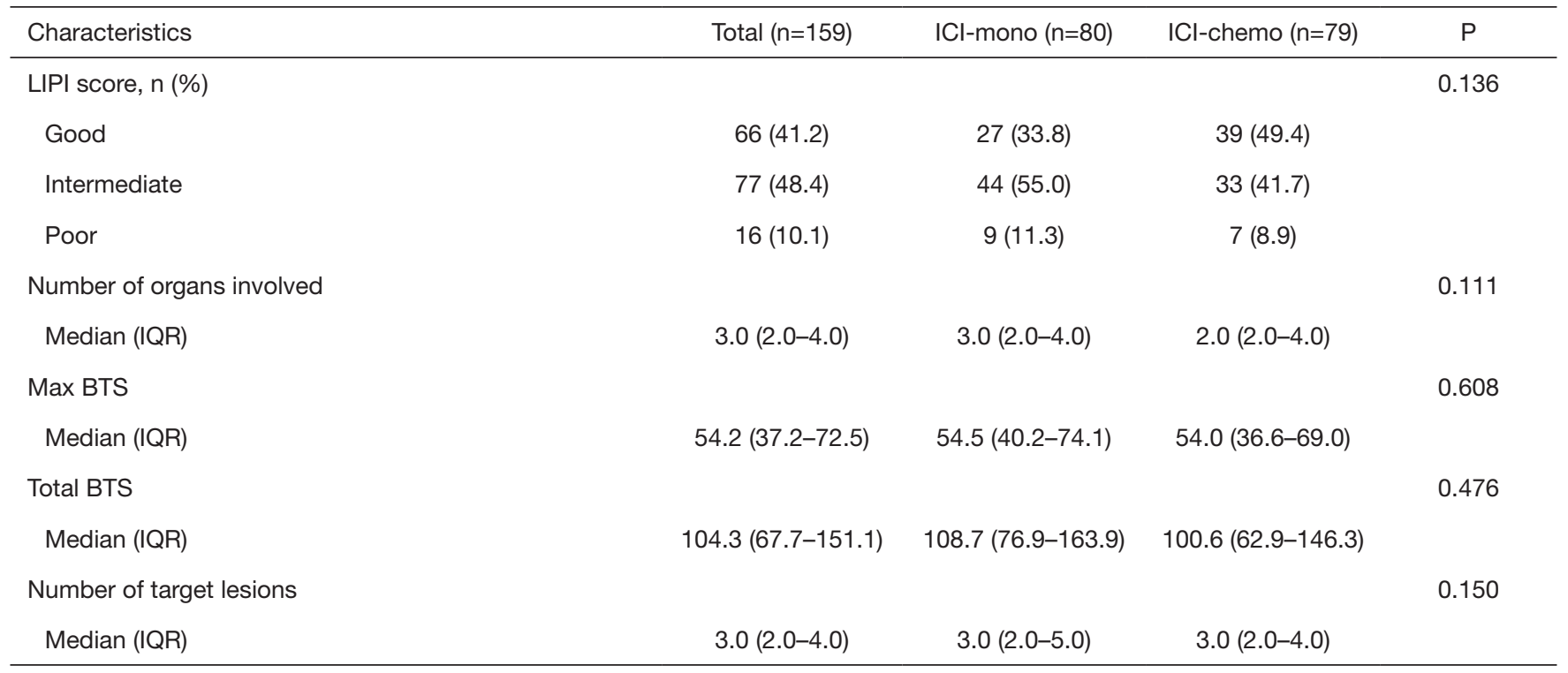

$\mathrm{ICl}$, immune checkpoint inhibitor; ICl-mono, ICI monotherapy; ICl-chemo, ICl in combination with chemotherapy; ECOG-PS, Eastern Cooperative Oncology Group performance status; NSCLC, non-small cell lung cancer; NOS, not otherwise specified; PD-L1, programmed death ligand-1; LIPI, lung immune prognostic index; IQR, interquartile range; BTS, baseline tumor size; max BTS, maximum BTS.

value in using the max BTS with PFS in patients who received ICI-mono was $47.76 \mathrm{~mm}$, which divided the patients into a small max BTS group $[\leq 47.76 \mathrm{~mm}$; 30 patients $(37.5 \%)]$ and a large max BTS group [>47.76 mm; 50 patients (62.5\%)] (Figure S2). The only significant difference between the small and large max BTS groups in patients who received ICI-mono was their LIPI scores (Table S2).

The cutoff value in using the total BTS with PFS in patients who received ICI-mono was $147.36 \mathrm{~mm}$, which classified the patients into a small total BTS group [ $\leq 147.36 \mathrm{~mm} ; 54$ patients $(67.5 \%)]$ and a large total BTS group [ $>147.36 \mathrm{~mm} ; 26$ patients $(32.5 \%)]$. The cutoff value in using the number of organs involved with PFS in patients who received ICI-mono was 4, which divided the patients into a small number of organs involved group $[\leq 4$; 67 patients $(83.4 \%)$ ] and a large number of organs involved group [>4; 13 patients $(16.3 \%)]$.

The optimal cutoff value in using the max BTS with PFS in patients who received ICI-chemo was $67.9 \mathrm{~mm}$, which divided the patients into a small max BTS group [ $\leq 67.9 \mathrm{~mm} ; 58$ patients $(73.4 \%)]$ and a large max BTS group [ $>67.9 \mathrm{~mm} ; 21$ patients $(26.6 \%)]$ [Figure S3]. The only significant differences between the small and large max BTS groups in patients who received ICI-chemo were their sex and LIPI scores (Table S3). The cutoff value in using the number of organs involved with PFS in patients who received ICI-chemo was $169.19 \mathrm{~mm}$, which classified the patients into a small total BTS group [ $\leq 169.19 \mathrm{~mm} ; 68$ patients $(86.1 \%)]$ and a large total BTS group [ $>169.19 \mathrm{~mm} ; 11$ patients $(13.9 \%)]$. The cutoff value in using the number of organs involved with PFS in patients who received ICI-chemo was 2 , which divided the patients into a small number of organs involved group $[\leq 2$; 44 patients $(55.7 \%)]$ and a large number of organs involved group [ $>2 ; 35$ patients $(44.3 \%)]$.

\section{Impact of the max BTS on the PFS, OS, and CBR of patients who received ICI-mono}

The median follow-up period for the censored cases was 12.6 (range, 0.2-44.7) months. The median PFS was 16.7 [95\% CI: 10.7-not available (NA)] months in the small max BTS group and 3.3 (95\% CI: 1.4-9.9) months in the large max BTS group, with the former having a significantly better PFS (HR, 0.39; 95\% CI: $0.22-0.70$; $\mathrm{P}=0.0012$; Figure $1 A$ ). The median PFS was 11.8 (95\% CI: 9.9-25.5) months in the small total BTS group and 2.4 (95\% CI: 1.2-8.3) months in the large total BTS group, with the former having a significantly better PFS (HR, 0.38; 95\% CI: $0.22-0.65 ; \mathrm{P}<0.001$; Figure $1 B)$. The median PFS was 10.7 (95\% CI: $5.8-16.7$ ) months in the small number of 

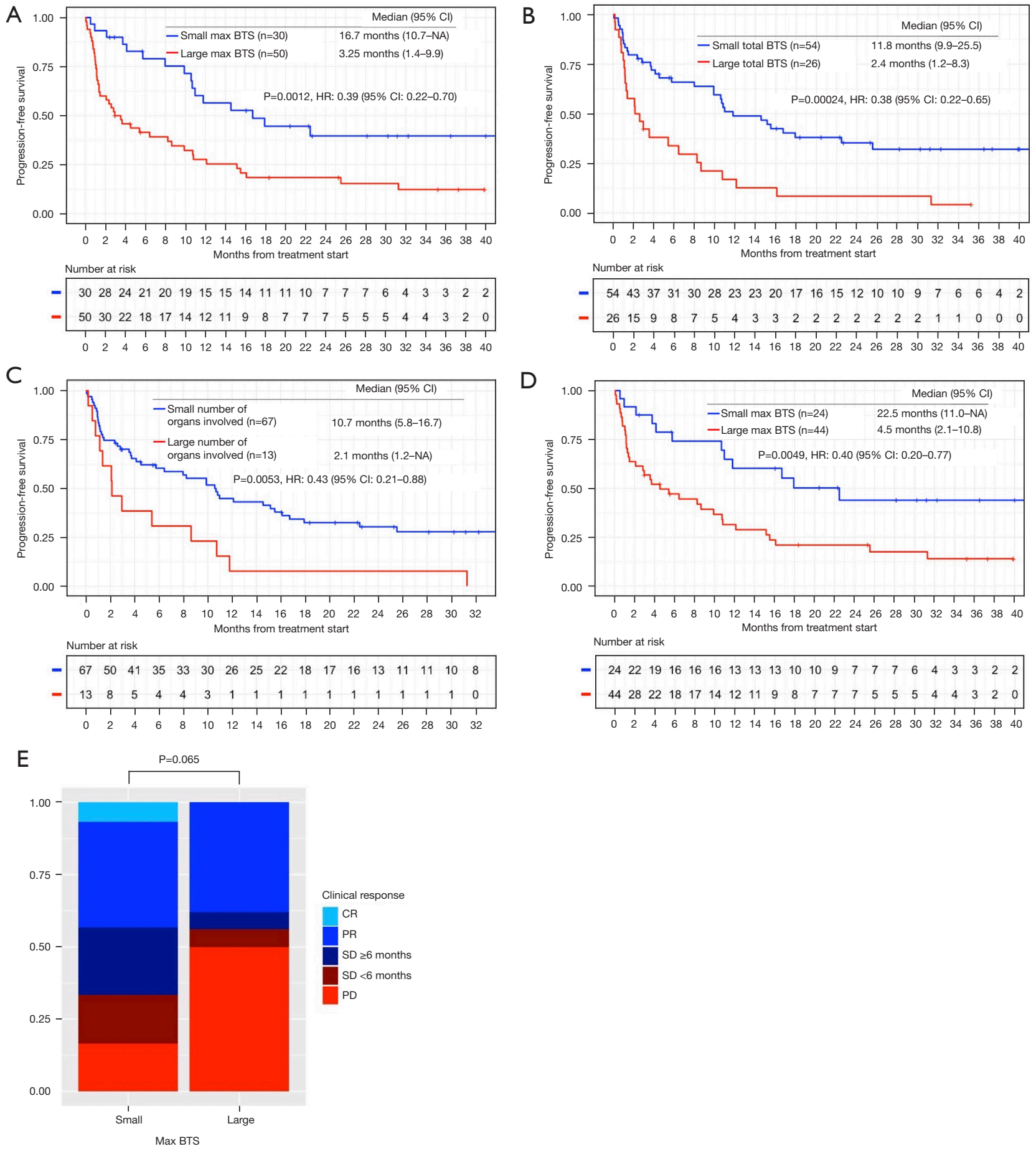

Figure 1 Clinical outcome of ICI-mono. (A-D) Kaplan-Meier estimates of the PFS of patients who received ICI-mono. (A) Comparison between the small and large max BTS groups (n=80). (B) Comparison between the small and large total BTS groups (n=80). (C) Comparison between the small and large number of organs involved groups ( $\mathrm{n}=80)$. (D) Comparison between the small and large max BTS groups with PD-L1 score $\geq 50 \%$ ( $n=68$ ). (E) Bar graph showing the percentage of patients with CR, PR SD $\geq 6$ months, SD $<6$ months, PD. Comparison between the small and large total BTS groups $(\mathrm{n}=80)$. ICI, immune checkpoint inhibitor; ICI-mono, ICI monotherapy; PFS, progressionfree survival; BTS, baseline tumor size; max BTS, maximum BTS; PD-L1, programmed death ligand-1; CR, complete response; PR, partial response; $\mathrm{SD}$, stable disease; $\mathrm{PD}$, progressive disease; $\mathrm{HR}$, hazard ratio; $\mathrm{CI}$, confidence interval. 
Table 2 Univariate and multivariable analysis of the PFS of patients treated with ICI-mono (n=80) or ICI-chemo (n=79)

\begin{tabular}{|c|c|c|c|c|c|c|}
\hline Variables & \multicolumn{3}{|c|}{ Univariate analysis } & \multicolumn{3}{|c|}{ Multivariable analysis $^{1}$} \\
\hline \multicolumn{7}{|l|}{$\mathrm{ICl}$-mono $(\mathrm{n}=80)$} \\
\hline Max BTS (small vs. large) & 0.39 & $0.22-0.70$ & 0.001 & 0.43 & $0.23-0.81$ & 0.009 \\
\hline Number of organs involved (small vs. large) & 0.43 & $0.21-0.88$ & 0.005 & 0.58 & $0.30-1.14$ & 0.112 \\
\hline Histological types (non-squamous vs. squamous) & 0.61 & $0.34-1.09$ & 0.096 & 0.78 & $0.43-1.41$ & 0.415 \\
\hline LIPI score (good vs. other) & 0.51 & $0.29-0.92$ & 0.025 & 0.61 & $0.32-1.17$ & 0.136 \\
\hline PD-L1 TPS ( $\geq 50 \%$ vs. other) & 0.58 & $0.29-1.18$ & 0.133 & 0.37 & $0.18-0.78$ & 0.008 \\
\hline Sex (male vs. female) & 0.95 & $0.50-1.85$ & 0.901 & NA & NA & NA \\
\hline \multicolumn{7}{|l|}{ ICl-chemo $(n=79)$} \\
\hline Max BTS (small vs. large) & 0.67 & $0.35-1.27$ & 0.212 & 0.58 & $0.29-1.18$ & 0.132 \\
\hline Number of organs involved (small vs. large) & 0.60 & $0.35-1.03$ & 0.064 & 0.48 & $0.28-0.83$ & 0.009 \\
\hline Histological types (non-squamous vs. squamous) & 0.59 & $0.32-1.09$ & 0.093 & 0.55 & $0.28-1.08$ & 0.079 \\
\hline PD-L1 TPS ( $\geq 50 \%$ vs. other) & 0.42 & $0.20-0.90$ & 0.025 & 0.38 & $0.17-0.82$ & 0.013 \\
\hline Sex (male vs. female) & 0.64 & $0.33-1.26$ & 0.197 & 0.65 & $0.32-1.32$ & 0.232 \\
\hline LIPI score (good vs. other) & 0.91 & $0.53-1.57$ & 0.730 & NA & NA & NA \\
\hline ECOG-PS (<1 vs. $\geq 1)$ & 0.78 & $0.43-1.42$ & 0.411 & NA & NA & NA \\
\hline
\end{tabular}

${ }^{1}$, max BTS, PD-L1 TPS, variables with $\mathrm{P}<0.20$ in the univariate analysis were included. $I C I$, immune checkpoint inhibitor; $I C l-m o n o, ~ I C l$ monotherapy; ICl-chemo, ICl in combination with chemotherapy; BTS, baseline tumor size; max BTS, maximum BTS; ECOG-PS, Eastern Cooperative Oncology Group performance status; LIPI, lung immune prognostic index; PD-L1, programmed death ligand-1; TPS, tumor proportion score; HR, hazard ratio; Cl, confidence interval; NA, not available.

organs involved group and 2.1 (95\% CI: 1.2-NA) months in the large number of organs involved group, with the former having a significantly better PFS (HR, 0.43; 95\% CI: $0.21-0.88 ; \mathrm{P}=0.0053$; Figure $1 C$ ). In patients with $\mathrm{PD}$ L1 TPS $\geq 50 \%$, the median PFS was 22.5 (95\% CI: 11.0 NA) months in the small max BTS group and 4.5 (95\% CI: 2.1-10.8) months in the large max BTS group, with the former having a significantly better PFS (HR, 0.40; $95 \%$ CI: 0.20-0.77; P=0.0049; Figure 1D).

The median OS was not reached in the small max BTS group, but it was 12.1 (95\% CI: 9.0-25.5) months in the large max BTS group, with the former having a significantly better OS (HR, 0.39; 95\% CI: 0.19-0.78; P=0.0065; Figure S4).

The multivariable analysis revealed that a better PFS was independently associated with the small max BTS (HR, 0.43; 95\% CI: $0.23-0.81 ; \mathrm{P}=0.009)$ and PD-L1 TPS $\geq 50 \%$ (HR, 0.37 ; 95\% CI: $0.18-0.78 ; \mathrm{P}=0.008$; Table 2).

The CBR tended to be higher in the small max BTS group than in the large max BTS group $(67.7 \%$ vs. $44.0 \%$; $\mathrm{P}=0.065$; Figure $1 E$ ). The disease control rate (DCR) was significantly greater in the small max BTS group than in the large max BTS group $(83.4 \%$ vs. $50.0 \% ; \mathrm{P}=0.004$; Table S4). 
Impact of the max BTS on the PFS, OS, and CBR of patients who received ICI-chemo

The median follow-up period for the censored cases was 13.5 (range, 0.3-28.4) months. The median PFS was 7.8 (95\% CI: 6.4-14.3) months in the small max BTS group and 15.6 (95\% CI: 6.3-NA) months in the large max BTS group. There was no significant difference in PFS between the small max BTS and large max BTS groups $(\mathrm{P}=0.21$; Figure 2A). The median PFS was 10.4 (95\% CI: 7.0-17.1) months in the small total BTS group and 4.4 (95\% CI: $3.0-$ NA) months in the large total BTS group. There was no significant difference in PFS between the small and large total BTS groups $(\mathrm{P}=0.062$; Figure $2 B)$. The median PFS was 11.5 (95\% CI: 7.8-19.9) months in the small number of organs involved group and 5.2 (95\% CI: 3.7-14.2) months in the large number of organs involved group. There was no significant difference in PFS between the small and large number of organs involved group $(\mathrm{P}=0.064$; Figure $2 C)$. In patients with PD-L1 TPS $\geq 50 \%$, the median PFS was 17.1 (95\% CI: 10.1-NA) months in the small max BTS group and 15.7 (95\% CI: 7.7-NA) months in the large max BTS group. There was no significant difference in PFS between the small and large max BTS groups in patients with a PDL1 TPS $\geq 50 \%$ ( $\mathrm{P}=0.74$; Figure $2 D)$.

The median OS was 26.0 (95\% CI: 16.6-NA) months in the small max BTS group, while it was not reached in the large max BTS group. There was no significant difference in the OS of the small and large max BTS groups $(\mathrm{P}=0.82$; Figure S5).

The multivariable analysis revealed that a better PFS was independently associated with a small number of organs involved (HR, 0.48; 95\% CI: 0.28-0.83; $\mathrm{P}=0.009)$ and PDL1 TPS $\geq 50 \%$ (HR, 0.38; 95\% CI: $0.17-0.82 ; \mathrm{P}=0.013$, Table 2).

There was no significant difference in CBR between the small and large max BTS groups $(77.6 \%$ vs. $80.1 \% ; \mathrm{P}=1.00)$ (Figure $2 E$ ). There was also no significant difference in DCR between the small and large max BTS groups (DCR, $82.8 \%$ vs. $90.5 \% ; \mathrm{P}=0.494$ ) (Table S5).

\section{Survival analysis of ICI-mono and ICI-chemo among patients with max $B T S \geq 50 \mathrm{~mm}$}

The median follow-up period for the censored cases was 12.5 (range, 0.2-40.0) months. The median PFS was 3.6 (95\% CI: 1.4-9.9) months in the ICI-mono group and 10.6 (95\% CI: 6.5-15.7) months in the ICI-chemo group, with the ICI-chemo group having a significantly better PFS (HR, 0.59; 95\% CI: 0.36-0.96; P=0.032; Figure $3 A$ ). The median OS was 15.2 (95\% CI: 9.3-25.5) months in the ICI-mono group, while it was not reached in the ICI-chemo group. OS tended to be longer in the ICI-chemo group than in the ICI-mono group (HR, 0.56; 95\% CI: 0.31-1.04; $\mathrm{P}=0.065$; Figure $3 B$ ). The ICI-chemo group had significantly longer RMST than the ICI-mono group (cutoff time $=12$ months, 10.16 vs. 8.36 months, $\mathrm{P}=0.037$; cutoff time $=18$ months, 14.12 vs. 11.25 months, $\mathrm{P}=0.039$; Figure $\mathrm{S} 6$ ).

In patients with a PD-L1 TPS $\geq 50 \%$, the median PFS was 4.5 (95\% CI: 2.1-10.8) months in the ICI-mono group and 15.7 (95\% CI: 7.7-NA) months in the ICI-chemo group. PFS tended to be longer in the ICI-chemo group than in the ICI-mono group in patients with a PD-L1 TPS $\geq 50 \%$ (HR, 0.38; 95\% CI: 0.14-1.10; $\mathrm{P}=0.064$; Figure 3C). In patients with a PD-L1 TPS $\geq 50 \%$, the median OS was 15.6 (95\% CI: 9.3-34.7) months in the ICI-mono group, while it was not reached in the ICI-chemo group. There was no significant difference in OS between the ICI-mono and ICI-chemo groups in patients with a PD-L1 TPS $\geq 50 \%(\mathrm{P}=0.32$; Figure $3 \mathrm{D})$.

The multivariable analysis revealed that a better PFS was independently associated with ICI-chemo (HR, 0.26; 95\% CI: $0.11-0.64 ; \mathrm{P}=0.004$ ) and PD-L1 TPS $\geq 50 \%$ (HR, 0.28; 95\% CI: 0.11-0.66; P=0.004, Table 3).

\section{Survival analysis of ICI-mono and ICI-chemo among patients with max BTS $<50 \mathrm{~mm}$}

The median follow-up period for the censored cases was 14.5 (range, 0.3-44.7) months. The median PFS was 14.6 (95\% CI: 10.6-NA) months in the ICI-mono group and 7.6 (95\% CI: 4.8-19.6) months in the ICI-chemo group. PFS tended to be longer in the ICI-mono group than in the ICI-chemo group (HR, 0.56; 95\% CI: 0.30-1.03; $\mathrm{P}=0.057$; Figure $\mathrm{S7A})$. The median $\mathrm{OS}$ was not reached in the ICI-mono group, while it was 26.0 (95\% CI: 17.1-NA) months in the ICI-chemo group. There was no significant difference in OS between the ICI-mono and ICI-chemo groups ( $\mathrm{P}=0.75$; Figure $\mathrm{S} 7 \mathrm{~B})$.

In patients with a PD-L1 TPS $\geq 50 \%$, the median PFS was 17.9 (95\% CI: 11.0-NA) months in the ICI-mono group and 17.1 (95\% CI: 7.4-NA) months in the ICIchemo group. There was no significant difference in PFS between the ICI-mono and ICI-chemo groups $(\mathrm{P}=0.90$; Figure S7C). In patients with a PD-L1 TPS $\geq 50 \%$, the median OS was not reached in the ICI-mono group, while 

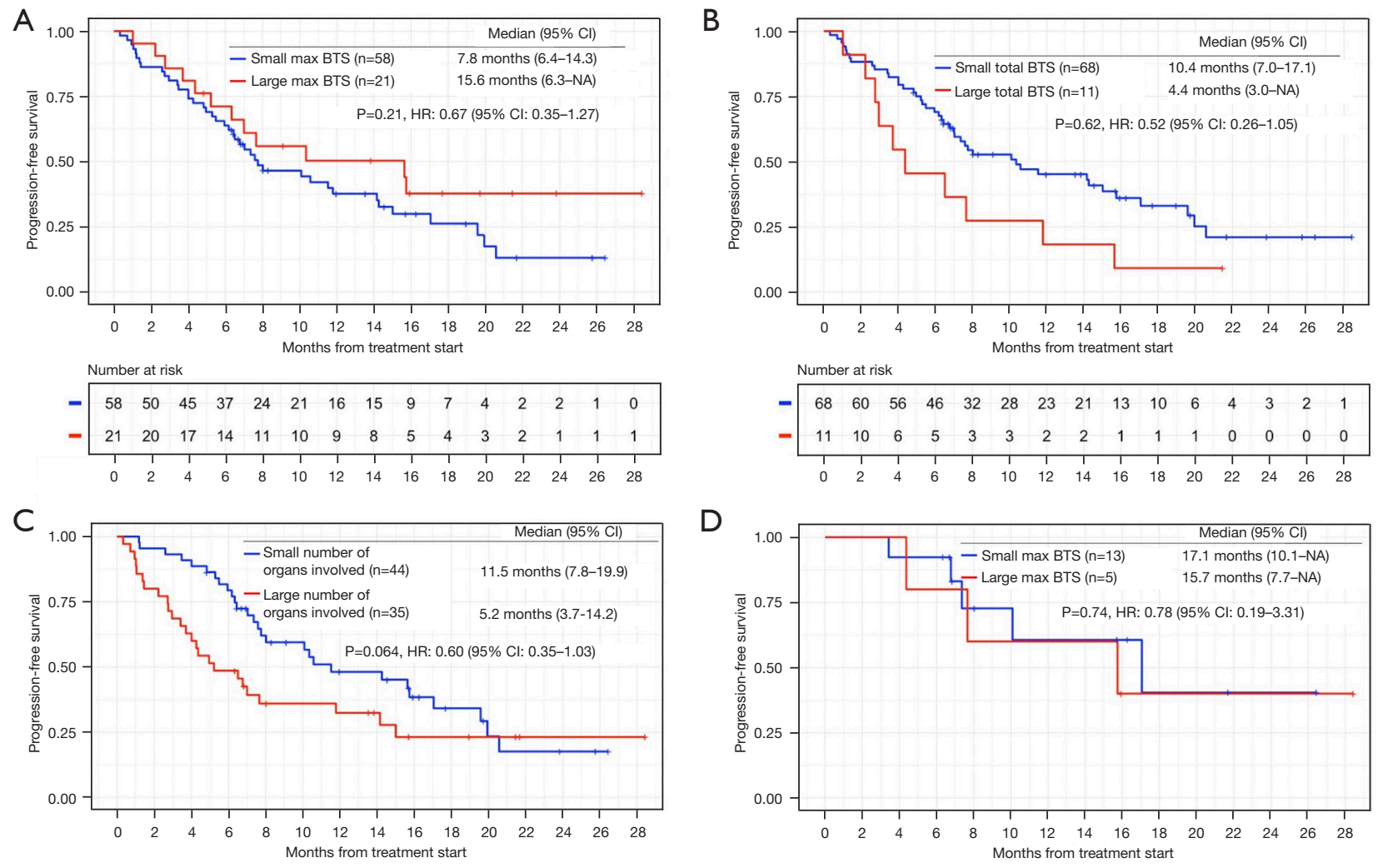

D
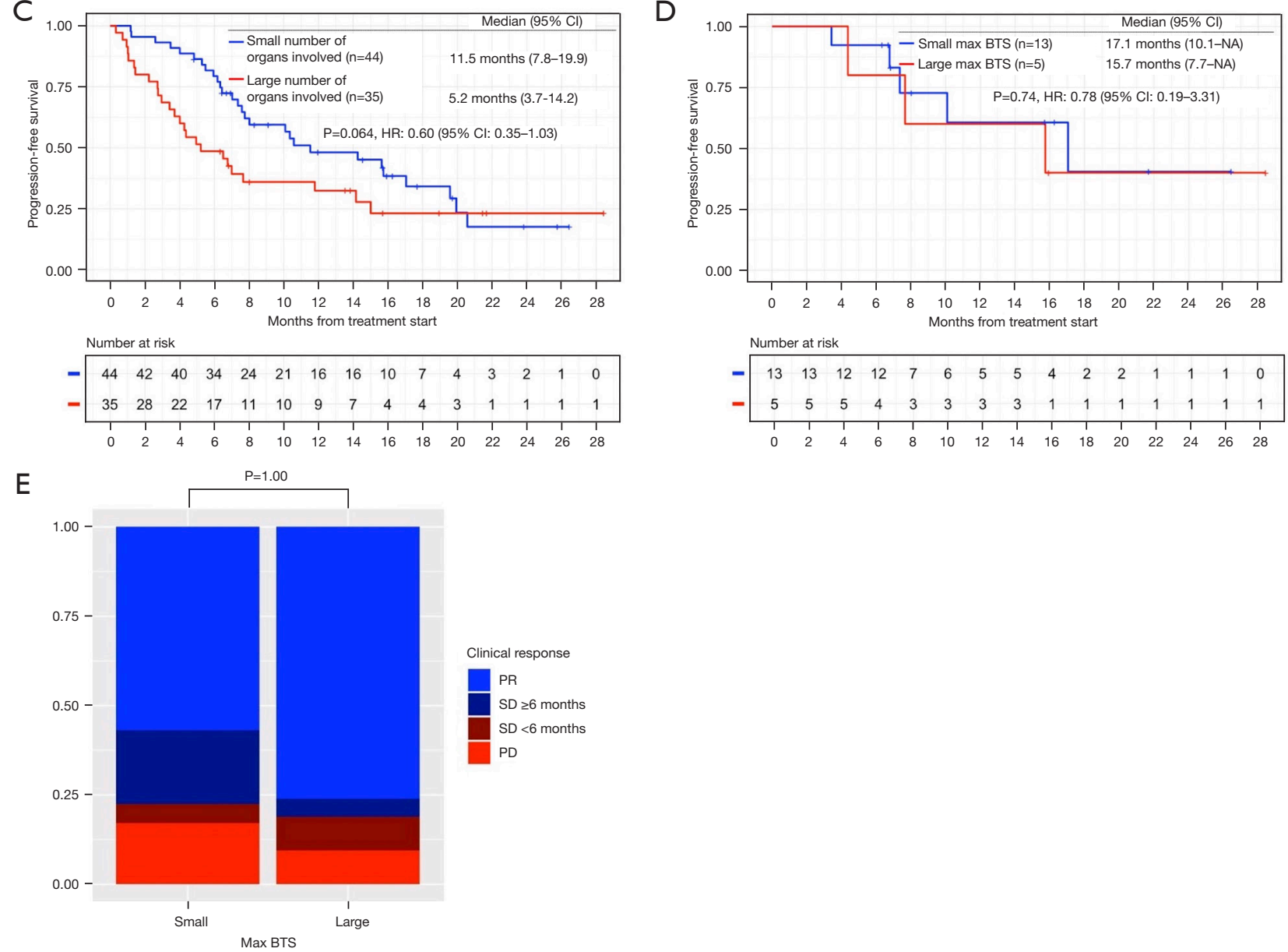

Figure 2 Clinical outcome of ICI-chemo. (A-D) Kaplan-Meier estimates of the PFS of patients who received ICI-chemo. (A) Comparison between the small and large max BTS groups $(\mathrm{n}=79)$. (B) Comparison between the small and large total BTS groups (n=79). (C) Comparison between the small and large number of organs involved groups ( $\mathrm{n}=79)$. (D) Comparison between the small and large max BTS groups with PD-L1 score $\geq 50 \%$ ( $n=18$ ). (E) Bar graph showing the percentage of patients with CR, PR SD $\geq 6$ months, SD $<6$ months, PD. Comparison between the small and large max BTS groups (n=79). ICI, immune-checkpoint inhibitor; ICI-chemo, ICI in combination with chemotherapy; PFS, progression-free survival; BTS, baseline tumor size; max BTS, maximum BTS; PD-L1, programmed death ligand-1; CR, complete response; PR, partial response; SD, stable disease; PD, progressive disease; HR, hazard ratio; CI, confidence interval. 

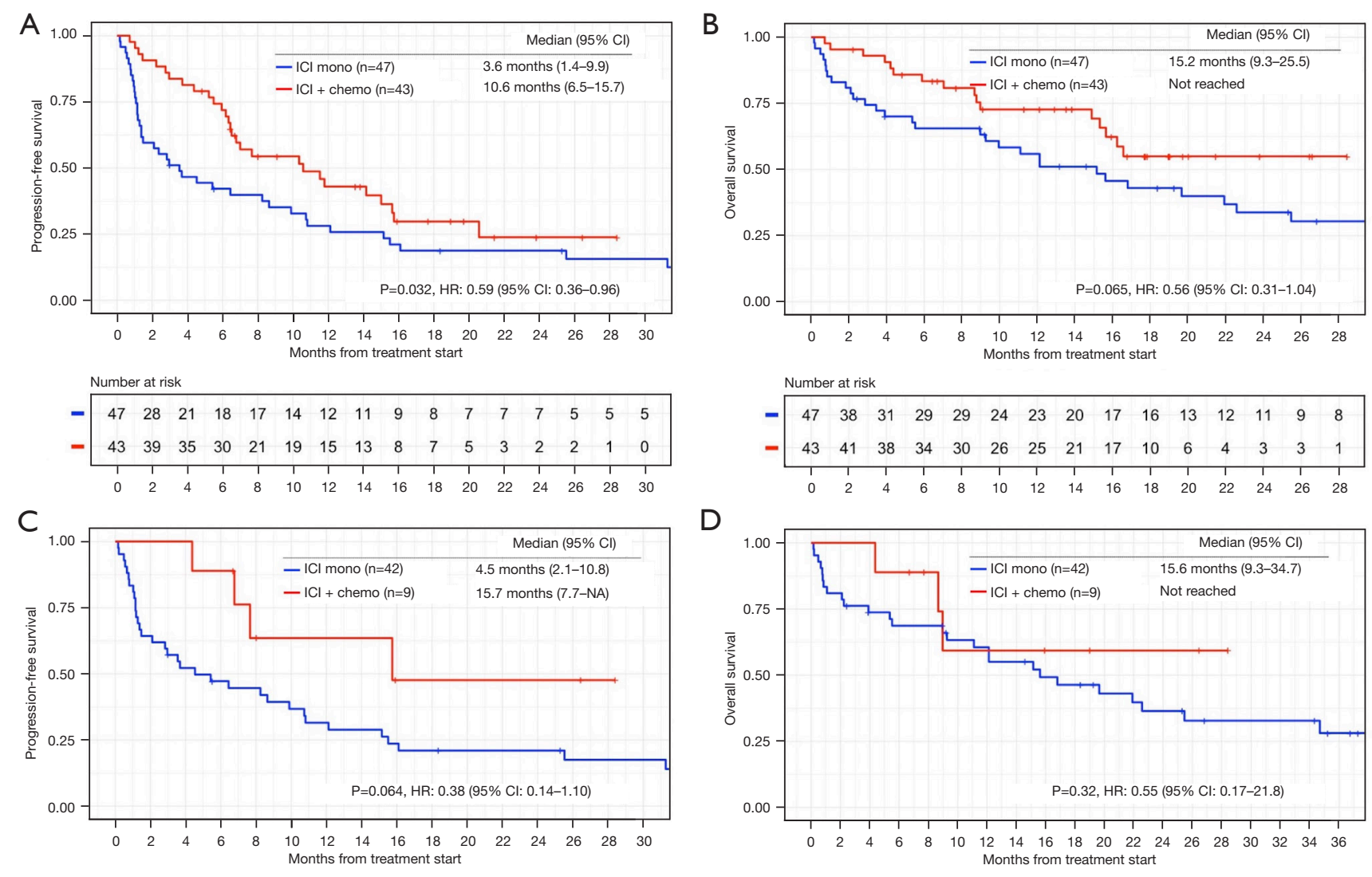

D
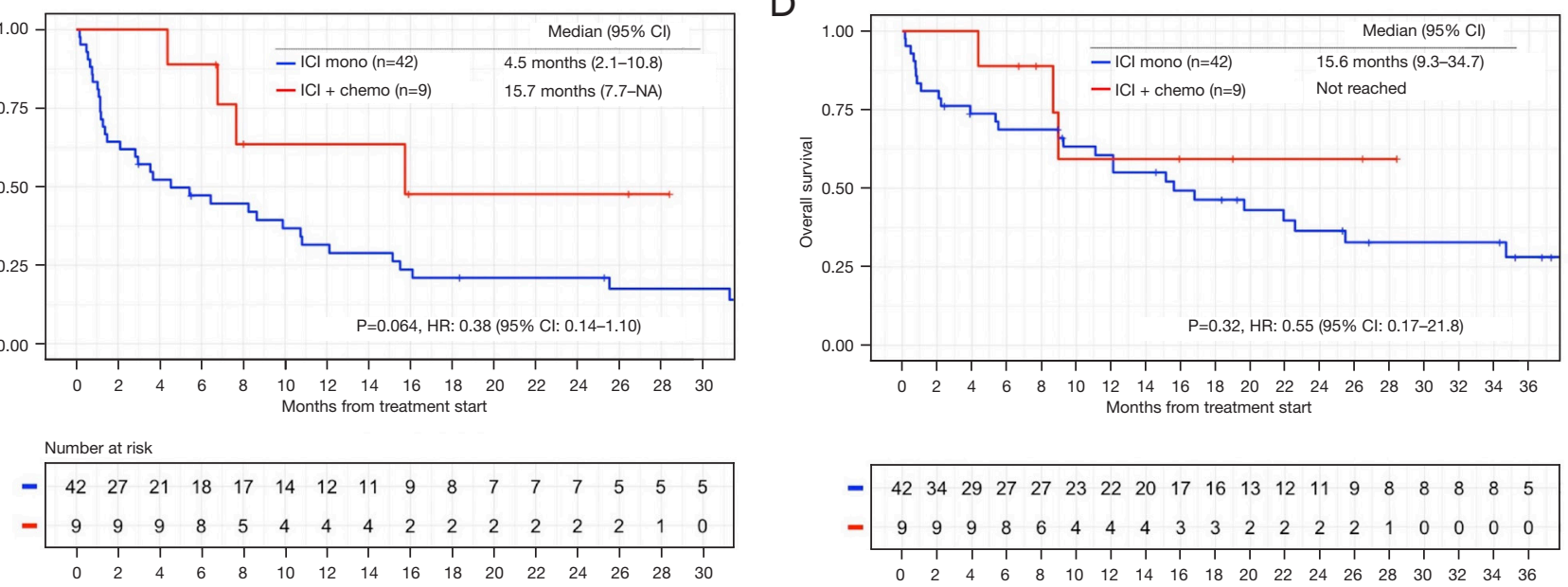

Figure 3 Comparison of the Kaplan-Meier estimates of patients with max BTS $\geq 50 \mathrm{~mm}$ who received ICI-mono and ICI-chemo. (A) PFS ( $\mathrm{n}=90)$. (B) OS (n=90). (C) PFS with PD-L1 score $\geq 50 \%$ group ( $\mathrm{n}=51)$. (D) OS with PD-L1 score $\geq 50 \%$ group (n=51). BTS, baseline tumor size; max BTS, maximum BTS; ICI, immune checkpoint inhibitor; ICI-mono, ICI monotherapy; ICI-chemo, ICI in combination with chemotherapy; PFS, progression-free survival; OS, overall survival; PD-L1, programmed death ligand-1; HR, hazard ratio; CI, confidence interval.

it was 17.1 (95\% CI: 17.1-NA) months in the ICI-chemo group. There was no significant difference in OS between the ICI-mono group and ICI-chemo group in patients with a PD-L1 TPS $\geq 50 \%(\mathrm{P}=0.71$; Figure $\mathrm{S} 7 \mathrm{D})$.

The multivariable analysis revealed that a better PFS was independently associated with histological types (HR, 0.23; 95\% CI: 0.26-0.50; $\mathrm{P}<0.001$; Table S6).

\section{Discussion}

To our knowledge, this is the first study to identify that the max BTS is a predictive factor of efficacy in patients with advanced NSCLC treated with first-line ICI-mono and
ICI-chemo. In patients treated with ICI-mono, the large max BTS was identified as an independent prognostic factor of worse PFS and OS, which was consistent with previous studies. By contrast, in patients treated with ICI-chemo, the large max BTS was not associated with worse PFS or OS.

This study mainly evaluated the association between the max BTS and patient outcomes. In several studies, the total BTS has been a surrogate marker for BTS to predict the efficacy of ICI $(5,7)$. However, evaluating the total BTS is time-consuming in everyday practice. The max BTS was reported to be another marker to assess BTS $(11,19)$. Instead of the total BTS, the max BTS was chosen to compare the ICI-mono group with the ICI-chemo group 
Table 3 Univariate and multivariable analysis of the PFS of patients with max BTS $\geq 50 \mathrm{~mm}(\mathrm{n}=90)$

\begin{tabular}{|c|c|c|c|c|c|c|}
\hline Variables & \multicolumn{3}{|c|}{ Univariate analysis } & \multicolumn{3}{|c|}{ Multivariable analysis $^{1}$} \\
\hline $\mathrm{ICl}$-chemo vs. ICI-mono & 0.59 & $0.36-0.96$ & 0.032 & 0.26 & $0.11-0.64$ & 0.004 \\
\hline Number of organs involved (<3 vs. $\geq 3$ ) & 0.62 & $0.38-1.03$ & 0.066 & 0.74 & $0.43-1.28$ & 0.282 \\
\hline PD-L1 TPS ( $\geq 50 \%$ vs. other) & 0.95 & $0.58-1.56$ & 0.850 & 0.28 & $0.11-0.66$ & 0.004 \\
\hline ECOG-PS (<2 vs. $\geq 2)$ & 0.58 & $0.34-1.00$ & 0.051 & 0.71 & $0.38-1.35$ & 0.300 \\
\hline Histological types (non-squamous vs. squamous) & 0.97 & $0.57-1.67$ & 0.910 & NA & NA & NA \\
\hline Sex (male vs. female) & 0.76 & $0.42-1.38$ & 0.372 & NA & NA & NA \\
\hline Age (<75 vs. $\geq 75)$ & 0.82 & $0.48-1.41$ & 0.477 & NA & NA & NA \\
\hline
\end{tabular}

${ }^{1}$, type of ICI treatment, PD-L1 TPS, variables with $\mathrm{P}<0.20$ in the univariate analysis were included. PFS, progression-free survival; BTS, baseline tumor size; max BTS, maximum BTS; ICl, immune checkpoint inhibitor; ICl-mono, ICl monotherapy; ICl-chemo, ICl in combination with chemotherapy; PD-L1, programmed death ligand-1; TPS, tumor proportion score; LIPI, lung immune prognostic index; ECOG-PS, Eastern Cooperative Oncology Group performance status; HR, hazard ratio; Cl, confidence interval; NA, not available.

because it only needs minimal effort to calculate the max BTS. Moreover, the simplicity of the max BTS reduces the interobserver discordance for the assessment, indicating its superiority over the total BTS. Furthermore, a good correlation between the max BTS and total BTS was confirmed in the ICI-mono and ICI-chemo groups (ICImono: coefficient, $0.735, \mathrm{P}<0.001$; ICI-chemo: coefficient 0.698, $\mathrm{P}<0.001$; Figure $\mathrm{S} 8$ ).

In the ICI-mono group, worse PFS, OS, and CBR were associated with large max BTS values, which aligned with prior studies $(7,11)$. The post hoc multivariate analysis showed that the total BTS $(>80 \mathrm{~mm})$ was a factor associated with worse OS in patients with previously treated NSCLC who received pembrolizumab (KEYNOTE-010) (20). A preclinical report explains the clinical failure of $\mathrm{PD}-1$ inhibitor against a melanoma with a larger tumor size, which resulted from an imbalance between T-cell reinvigoration and pretreatment tumor mass (21). Large tumor masses cause tissue hypoxia, inhibiting immune plasticity and promoting an immune-suppressive microenvironment (22). Moreover, a larger tumor volume correlates with plasmabased circulating tumor DNA, which was suggested as a prognostic clinical biomarker of ICI response (23).

In the ICI-chemo group, worse PFS, OS, and CBR were not associated with large max BTS values. Interestingly, a worse PFS was independently associated with a large number of organs involved. Their differences might be explained by the inclusion of the nontarget lesions.
The max BTS included one lesion, while the number of organs involved included the target and nontarget lesions. Therefore, adding chemotherapy to ICI could not overcome NSCLC with poor prognosis due to many metastatic organ sites (19). The post hoc analysis in randomized phase III studies (KEYNOTE-189, IMpower130) showed that brain/ liver metastases were poor prognostic factors in patients treated with ICI-chemo $(24,25)$. Moreover, liver metastases were reported to be associated with reduced $\mathrm{CT} 8^{+} \mathrm{T}$-cell infiltration (26). The large number of organs involved might reflect the presence of brain or liver metastases, which led to its association with worse PFS in the ICI-chemo group.

In patients with $\max$ BTS $\geq 50 \mathrm{~mm}$, the multivariable analysis showed that the choice of ICI-chemo vs. ICImono was an independent predictive factor for PFS. A similar result was observed in the group with PD-L1 TPS $\geq 50 \%$, but it was not statistically significant due to the small sample size. In patients with max BTS $<50 \mathrm{~mm}$, the choice of ICI-chemo $v s$. ICI-mono was not an independent predictive factor for PFS. Currently, in patients with PDL1 expression $\geq 50 \%$, pembrolizumab or atezolizumab monotherapy and chemoimmunotherapy can be used. Several retrospective studies focused on the comparison of ICI-chemo vs. ICI-mono but presented paradoxical results. Some retrospective studies showed the benefit of adding chemotherapy, while other real-world data demonstrated that adding chemotherapy had no benefit, except in a subgroup of patients with no smoking history $(13,27,28)$. 
These studies have not evaluated BTS as a stratification factor. In our study, these two groups were compared, choosing $50 \mathrm{~mm}$ of the max BTS as the cutoff value, which was close to the median number of all patients. Moreover, this value was consentient with a previous study and practical for use in a clinical setting (11). This is the first study to show that the max BTS was a predictive factor in patients who received ICI-mono $v s$. ICI-chemo, using 50 $\mathrm{mm}$ as the cutoff value. A similar trend was observed in PDL1 TPS $\geq 50 \%$, suggesting that it is better to use ICI-chemo for patients with PD-L1 TPS $\geq 50 \%$ and large max BTS. Moreover, these findings suggest the importance of BTS as a factor for further prospective studies on ICI combination, chemoradiotherapy, neoadjuvant ICI therapy, and adjuvant ICI therapy.

The association between the LIPI score and BTS was evaluated. Previous studies have suggested that the LIPI score was a prognostic marker of benefits from ICI-mono (2). It reflects an inflammatory status, which was a proposed mechanism of immunoresistance in patients with cancer. However, LIPI score could not be a prognostic marker of the treatment response to ICI-chemo, which was consistent with our cohort (29). In patients with max BTS $\geq 50 \mathrm{~mm}$, the univariable and multivariable analysis showed that the LIPI score appeared to be a prognostic factor for PFS, but it was not statistically significant. This might suggest that the immune system is still crucial in predicting the efficacy of ICI treatment aside from the tumor volume. Furthermore, the existence of groups with large max BTS values and good clinical outcomes in patients treated with ICI-mono might suggest the importance of the tumor microenvironment.

Notwithstanding the consistent evidence underpinning its promising prognostic and predictive factor, BTS is not commonly considered for enrolment in clinical trials, neither as an inclusion/exclusion criterion nor as a stratification factor. A post-hoc efficacy analysis of KEYNOTE-189 showed ICI-chemo showed consistent clinical benefit regardless of BTS (30). These findings suggest that BTS might be evaluated in future ICI trials and integrated into clinical practice to provide treatment expectations when choosing between ICI-mono vs. ICIchemo.

There are two ongoing trials that directly compare ICI-mono $v s$. ICI-chemo. In treatment-naive advanced NSCLC with PD-L 1 expression $\geq 1 \%$, INSIGNA (NCT 03793179), an ongoing randomized phase III study, compares the clinical outcomes of pembrolizumab monotherapy $v s$. pembrolizumab plus chemotherapy. In advanced NSCLC with PD-L1 expression $\geq 50 \%$, PERSEE (NCT04547504), an ongoing randomized phase III study, is evaluating pembrolizumab alone $v s$. pembrolizumab plus chemotherapy as a first-line treatment. Our findings would corroborate these ongoing prospective studies for the subgroup analysis.

Currently, ICI-mono, ICI-chemo, and dual ICI with or without chemotherapy can be used for tumors with PDL1 expression $\geq 50 \%$. Moreover, a tumor with PD-L1 expression $\geq 50 \%$ is not equal. Among patients with $\mathrm{PD}$ L1 expression $\geq 50 \%$, the clinical outcomes of patients with NSCLC with PD-L1 expression $\geq 90 \%$ were significantly improved with ICI-mono as a first-line treatment (31). Thus, adding chemotherapy to immunotherapy in PDL1 expression $\geq 90 \%$ might be another issue to investigate further. Further studies are warranted to validate the prognostic and predictive role of BTS and the clinical outcomes in these cohorts: patients treated with dual ICI with or without chemotherapy and patients with tumor PDL1 expression $\geq 90 \%$ treated with ICI-mono $v s$. ICI-chemo.

Because of its retrospective nature, the results of this study were hypothesis-generating rather than conclusive. First, the sample size was relatively small, especially in PD-L1 TPS $\geq 50 \%$ with lower statistical power. Although the baseline clinical characteristics of the patients in the ICI-mono and ICI-chemo groups were moderately wellbalanced, we acknowledged the existence of a selection bias that patients with aggressive status, younger age, better PS, and no significant medical comorbidities were more likely to receive ICI-chemo and patients with indolent status, older age, poorer PS, and many medical comorbidities were more likely to receive ICI-mono. The strong association of PD-L1 expression with clinical outcomes might confound our result; however, previous studies have shown that PDL1 expression has limitations as a predictive biomarker in NSCLC (1). Our evaluation of the max BTS with multivariate analysis, including number of organs involved, age, PS, and PD-L1 expression, could help minimize the potential for confounding bias. Second, there is no validation data set. Most of our cohort was men because we excluded the patients with driver mutations such as EGFR mutation, which was a common driver mutation among never-smoker Asian women. Although the recurrent at staging represented patients with widespread metastatic cancer or oligo-recurrence resistant to local treatment, the detail of their recurrent patterns was different for each patient. Third, the accurate tumor mass could not be 
directly evaluated. The bone and pleural metastatic lesions, multiple microtumors in one lesion, and multiple massive lesions were difficult to assess. Fourth, the follow-up time was relatively short to fully evaluate the long-term survival outcomes, but the early response might be related to a longer survival (3). Besides, PFS based on imaging metrics depends on the tumor assessment schedule and may not always parallel the clinical outcomes of OS and therapeutic efficacy. Fifth, the adverse events were not compared because of the incomplete records. Prospective larger trials with longer follow-up periods are needed to validate these findings.

\section{Conclusions}

The max BTS may have different predicting efficacy for patients with NSCLC treated with ICI-mono and ICIchemo. Our findings may provide a valuable and simple tool to differentiate patients who would benefit from ICI-chemo from those who would benefit from ICI-mono.

\section{Acknowledgments}

We thank Enago (https://www.enago.jp) for editing a draft of this manuscript.

Funding: None.

\section{Footnote}

Reporting Checklist: The authors have completed the STROBE reporting checklist. Available at https://tlcr. amegroups.com/article/view/10.21037/tlcr-21-815/rc

Data Sharing Statement: Available at https://tlcr.amegroups. com/article/view/10.21037/tlcr-21-815/dss

Peer Review File: Available at https://tlcr.amegroups.com/ article/view/10.21037/tlcr-21-815/prf

Conflicts of Interest: All authors have completed the ICMJE uniform disclosure form (available at https://tlcr. amegroups.com/article/view/10.21037/tlcr-21-815/coif). TH has received Payment for speakers' bureaus from Chugai Pharmaceutical, outside the submitted work. KW has received honoraria for speakers from AstraZeneca, Chugai Pharmaceutical, MSD, Taiho Pharmaceutical, Pfizer, Eli Lilly Japan, Boehringer Ingelheim, and Ono Pharmaceutical, outside the submitted work. SK received honoraria for lectures from AstraZeneca and Chugai Pharmaceutical, outside the submitted work. MY has received honoraria for speakers from Chugai Pharmaceutical, Ono Pharmaceutical, AstraZeneca, TAIHO Pharmaceutical, Takeda Pharmaceutical, Boehringer Ingelheim, and Pfizer, outside the submitted work. YH has received for speakers from AstraZeneca, Ono Pharmaceutical, and Chugai Pharmaceutical, outside the submitted work. The other authors have no conflicts of interest to declare.

Ethical Statement: The authors are accountable for all aspects of the work in ensuring that questions related to the accuracy or integrity of any part of the work are appropriately investigated and resolved. All procedures performed in this study involving human participants were in accordance with the Declaration of Helsinki (as revised in 2013). The study was approved by the institutional review boards of Tokyo Metropolitan Cancer and Infectious Diseases Center of Komagome Hospital (No. 2725). Individual consent for this retrospective analysis was waived.

Open Access Statement: This is an Open Access article distributed in accordance with the Creative Commons Attribution-NonCommercial-NoDerivs 4.0 International License (CC BY-NC-ND 4.0), which permits the noncommercial replication and distribution of the article with the strict proviso that no changes or edits are made and the original work is properly cited (including links to both the formal publication through the relevant DOI and the license). See: https://creativecommons.org/licenses/by-nc-nd/4.0/.

\section{References}

1. Brueckl WM, Ficker JH, Zeitler G. Clinically relevant prognostic and predictive markers for immune-checkpointinhibitor (ICI) therapy in non-small cell lung cancer (NSCLC). BMC Cancer 2020;20:1185.

2. Mezquita L, Auclin E, Ferrara R, et al. Association of the Lung Immune Prognostic Index With Immune Checkpoint Inhibitor Outcomes in Patients With Advanced Non-Small Cell Lung Cancer. JAMA Oncol 2018;4:351-7.

3. Remon J, Hendriks LEL, Besse B. Paving the Way for Long-Term Survival in Non-Small-Cell Lung Cancer. J Clin Oncol 2021;39:2321-3.

4. Joseph RW, Elassaiss-Schaap J, Kefford R, et al. Baseline Tumor Size Is an Independent Prognostic Factor for 
Overall Survival in Patients with Melanoma Treated with Pembrolizumab. Clin Cancer Res 2018;24:4960-7.

5. Inoue H, Yokota T, Hamauchi S, et al. Pre-treatment tumor size impacts on response to nivolumab in head and neck squamous cell carcinoma. Auris Nasus Larynx 2020;47:650-7.

6. Katsurada M, Nagano T, Tachihara M, et al. Baseline Tumor Size as a Predictive and Prognostic Factor of Immune Checkpoint Inhibitor Therapy for Non-small Cell Lung Cancer. Anticancer Res 2019;39:815-25.

7. Miyawaki T, Kenmotsu H, Mori K, et al. Association Between Clinical Tumor Burden and Efficacy of Immune Checkpoint Inhibitor Monotherapy for Advanced Non-Small-Cell Lung Cancer. Clin Lung Cancer 2020;21:e405-14.

8. Hopkins AM, Kichenadasse G, McKinnon RA, et al. Baseline tumor size and survival outcomes in lung cancer patients treated with immune checkpoint inhibitors. Semin Oncol 2019;46:380-4.

9. Sakata Y, Kawamura K, Ichikado K, et al. Comparisons between tumor burden and other prognostic factors that influence survival of patients with non-small cell lung cancer treated with immune checkpoint inhibitors. Thorac Cancer 2019;10:2259-66.

10. Friedlander P. The use of baseline tumor size to prognosticate overall survival in stage IV melanoma patients treated with the PD-1 inhibitor pembrolizumab. Ann Transl Med 2019;7:S24.

11. Hakozaki T, Hosomi Y, Kitadai R, et al. Efficacy of immune checkpoint inhibitor monotherapy for patients with massive non-small-cell lung cancer. J Cancer Res Clin Oncol 2020;146:2957-66.

12. Ferrara R, Mezquita L, Texier M, et al. Hyperprogressive Disease in Patients With Advanced Non-Small Cell Lung Cancer Treated With PD-1/PD-L1 Inhibitors or With Single-Agent Chemotherapy. JAMA Oncol 2018;4:1543-52.

13. Chen Y, Wang Y, Yang Z, et al. Pembrolizumab Alone or Combined With Chemotherapy in Advanced NSCLC With PD-L1 $\geq 50 \%$ : Results of a Retrospective Study. Front Oncol 2021;11:691519.

14. Kim R, Keam B, Hahn S, et al. First-line Pembrolizumab Versus Pembrolizumab Plus Chemotherapy Versus Chemotherapy Alone in Non-small-cell Lung Cancer: A Systematic Review and Network Meta-analysis. Clin Lung Cancer 2019;20:331-338.e4.

15. Liang H, Lin G, Wang W, et al. Feasibility and safety of PD-1/L1 inhibitors for non-small cell lung cancer in front-line treatment: a Bayesian network meta-analysis. Transl Lung Cancer Res 2020;9:188-203.

16. Liu J, Li C, Seery S, et al. Identifying optimal first-line interventions for advanced non-small cell lung carcinoma according to PD-L1 expression: a systematic review and network meta-analysis. Oncoimmunology 2020;9:1746112.

17. Chen Y, Huang J, He X, et al. A novel approach to determine two optimal cut-points of a continuous predictor with a $\mathrm{U}$-shaped relationship to hazard ratio in survival data: simulation and application. BMC Med Res Methodol 2019;19:96.

18. Tian L, Zhao L, Wei LJ. Predicting the restricted mean event time with the subject's baseline covariates in survival analysis. Biostatistics 2014;15:222-33.

19. Oh Y, Taylor S, Bekele BN, et al. Number of metastatic sites is a strong predictor of survival in patients with nonsmall cell lung cancer with or without brain metastases. Cancer 2009;115:2930-8.

20. Herbst RS, Baas P, Kim DW, et al. Factors associated with better overall survival (OS) in patients with previously treated, PD-L1-expressing, advanced NSCLC: Multivariate analysis of KEYNOTE-010. J Clin Oncol 2017;35:abstr 9090.

21. Huang AC, Postow MA, Orlowski RJ, et al. T-cell invigoration to tumour burden ratio associated with antiPD-1 response. Nature 2017;545:60-5.

22. Chouaib S, Noman MZ, Kosmatopoulos K, et al. Hypoxic stress: obstacles and opportunities for innovative immunotherapy of cancer. Oncogene 2017;36:439-45.

23. Lam VK, Zhang J, Wu CC, et al. Genotype-Specific Differences in Circulating Tumor DNA Levels in Advanced NSCLC. J Thorac Oncol 2021;16:601-9.

24. Gadgeel S, Rodríguez-Abreu D, Speranza G, et al. Updated Analysis From KEYNOTE-189: Pembrolizumab or Placebo Plus Pemetrexed and Platinum for Previously Untreated Metastatic Nonsquamous Non-Small-Cell Lung Cancer. J Clin Oncol 2020;38:1505-17.

25. West H, McCleod M, Hussein M, et al. Atezolizumab in combination with carboplatin plus nab-paclitaxel chemotherapy compared with chemotherapy alone as firstline treatment for metastatic non-squamous non-smallcell lung cancer (IMpower130): a multicentre, randomised, open-label, phase 3 trial. Lancet Oncol 2019;20:924-37.

26. Tumeh PC, Hellmann MD, Hamid O, et al. Liver Metastasis and Treatment Outcome with Anti-PD-1 Monoclonal Antibody in Patients with Melanoma and NSCLC. Cancer Immunol Res 2017;5:417-24.

27. Peters S, Dafni U, Perol M, et al. VP2-2021: Effectiveness 
of PD-(L) 1 inhibitors alone or in combination with platinum-doublet chemotherapy in first-line (1L) nonsquamous non-small cell lung cancer (Nsq-NSCLC) with high PD-L1 expression using real-world data. Ann Oncol 2021;32:687-8.

28. Han B, Zhang B, Hu M, et al. 111P Advanced non-small cell lung cancer with PD-L1 $\geq 50 \%$ : Pembrolizumab alone or combined with chemotherapy. J Thorac Oncol 2021;16:S758-9..

29. Wang W, Huang Z, Yu Z, et al. Prognostic Value of the Lung Immune Prognostic Index May Differ in Patients Treated With Immune Checkpoint Inhibitor Monotherapy or Combined With Chemotherapy for Non-small Cell Lung Cancer. Front Oncol 2020;10:572853.

30. Gadgeel S, Grey J, Rizzo MT, et al. Pemetrexed and platinum plus pembrolizumab in patients with metastatic non-squamous non-small cell lung cancer by tumor burden at baseline: A post-hoc efficacy analysis of KEYNOTE-189. Cancer Res 2021;81:abstr 442.

31. Aguilar EJ, Ricciuti B, Gainor JF, et al. Outcomes to first-line pembrolizumab in patients with non-small-cell lung cancer and very high PD-L1 expression. Ann Oncol 2019;30:1653-9.

Cite this article as: Uehara Y, Hakozaki T, Kitadai R, Narita K, Watanabe K, Hashimoto K, Kawai S, Yomota M, Hosomi Y. Association between the baseline tumor size and outcomes of patients with non-small cell lung cancer treated with first-line immune checkpoint inhibitor monotherapy or in combination with chemotherapy. Transl Lung Cancer Res 2022;11(2):135149. doi: $10.21037 /$ tlcr-21-815 


\section{Patient flow diagram}

Patients with advanced or recurrence non-small cell lung cancer
received first-line PD-1/ PD-L1 inhibitors at Komagome Hospital
Date: January 2016 and April $2021(\mathrm{n}=191)$
Excluded for recurrence after chemoradiotherapy $(\mathrm{n}=23)$
$\begin{aligned} & \text { Excluded for EGFR/ALK /ROS1 /BRAF positive }(\mathrm{n}=1) \\ & \text { Expluded for clinical trials patients who received } \\ & \text { No target lesions }(\mathrm{n}=1) \\ & \text { Hospital transfer before first evaluation }(\mathrm{n}=1)\end{aligned}$
Patients included in this study $(\mathrm{n}=159)$

Figure S1 Study flowchart. PD-1, programmed death-1; PD-L1, programmed death ligand-1.

Table S1 Site of the largest target lesions

\begin{tabular}{|c|c|c|c|}
\hline Site of the largest target lesions & ICl-mono (n=80), n (\%) & ICl-chemo ( $\mathrm{n}=79), \mathrm{n}(\%)$ & $\mathrm{P}$ \\
\hline Bone & $4(5.0)$ & $7(8.9)$ & - \\
\hline Intrathoracic lymph node & $3(3.7)$ & $5(6.3)$ & - \\
\hline Pleura & $6(7.4)$ & $2(2.5)$ & - \\
\hline Adrenal gland & $4(5.0)$ & $2(2.5)$ & - \\
\hline Lung (metastasis) & $0(0.0)$ & $2(2.5)$ & - \\
\hline Others & $4(5.0)$ & $2(2.5)$ & - \\
\hline Liver & $0(0.0)$ & $1(1.3)$ & - \\
\hline
\end{tabular}

$\mathrm{ICl}$, immune checkpoint inhibitor; ICl-mono, ICl monotherapy; ICl-chemo, ICl in combination with chemotherapy. 


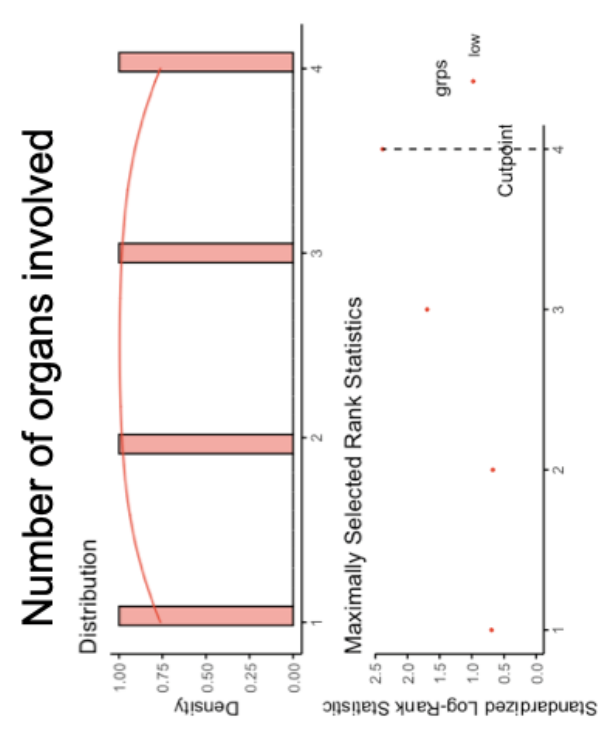

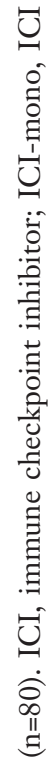

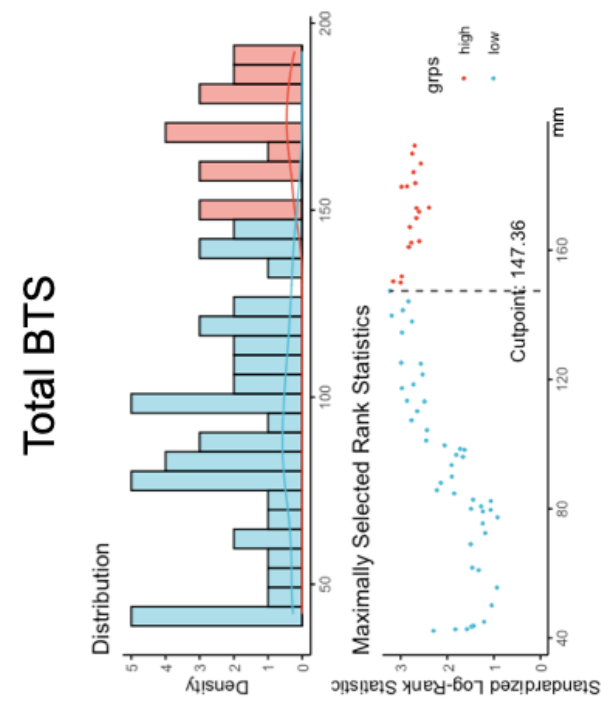

范

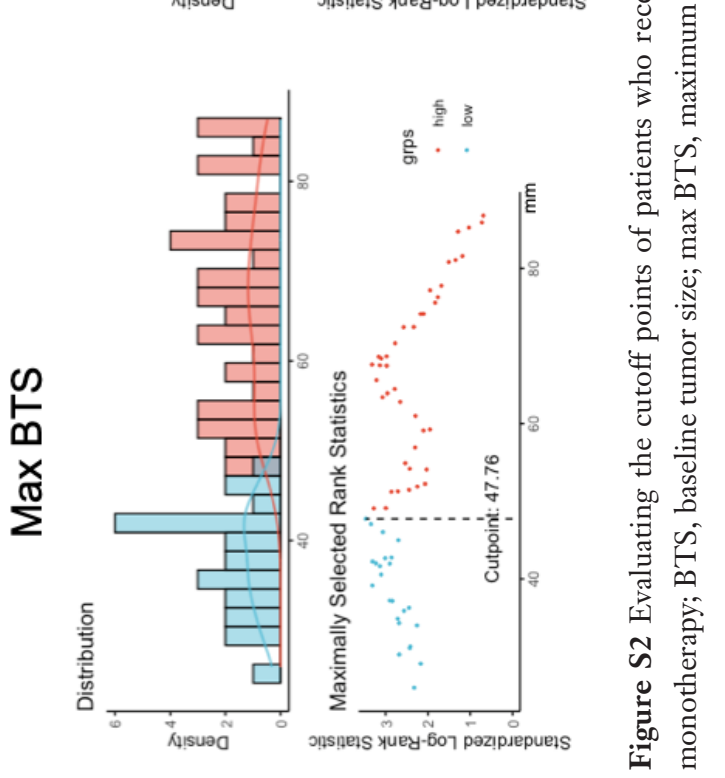


Table S2 Characteristics of patients who received ICI-mono stratified by max BTS ( $\mathrm{n}=80)$

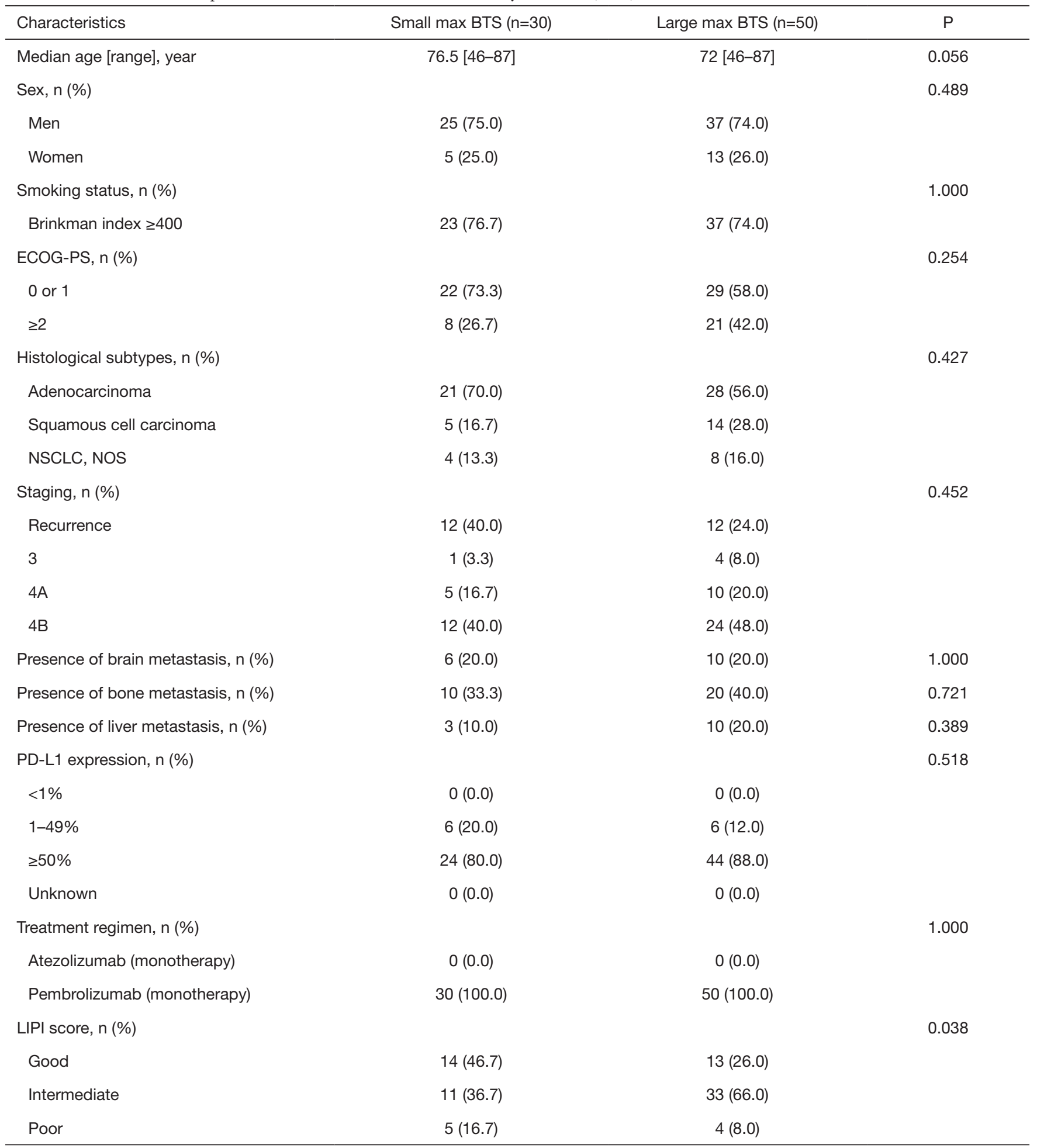

$\mathrm{ICl}$, immune checkpoint inhibitor; ICl-mono, ICI monotherapy; BTS, baseline tumor size; max BTS, maximum BTS; ECOG-PS, Eastern Cooperative Oncology Group performance status; NSCLC, non-small cell lung cancer; NOS, not otherwise specified; PD-L1, programmed death ligand-1; LIPI, lung immune prognostic index. 


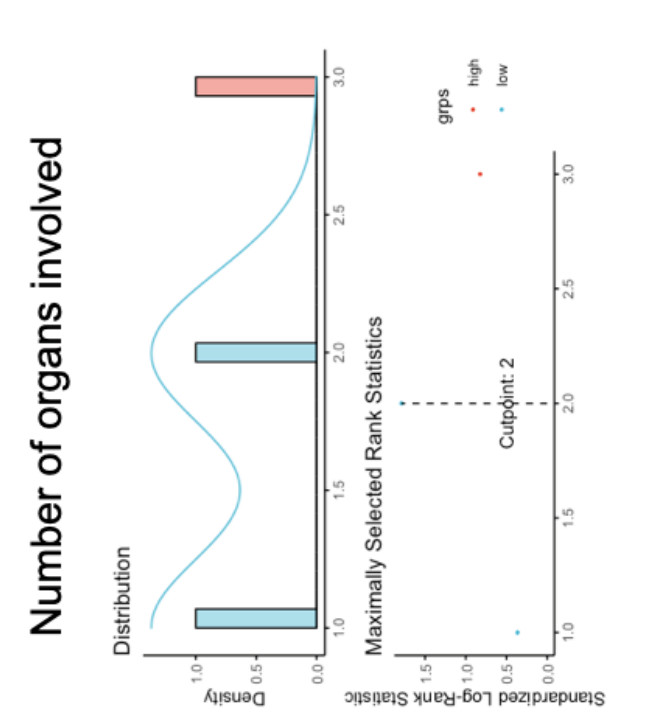

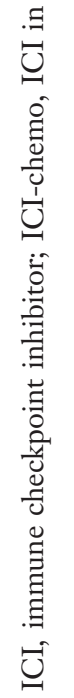
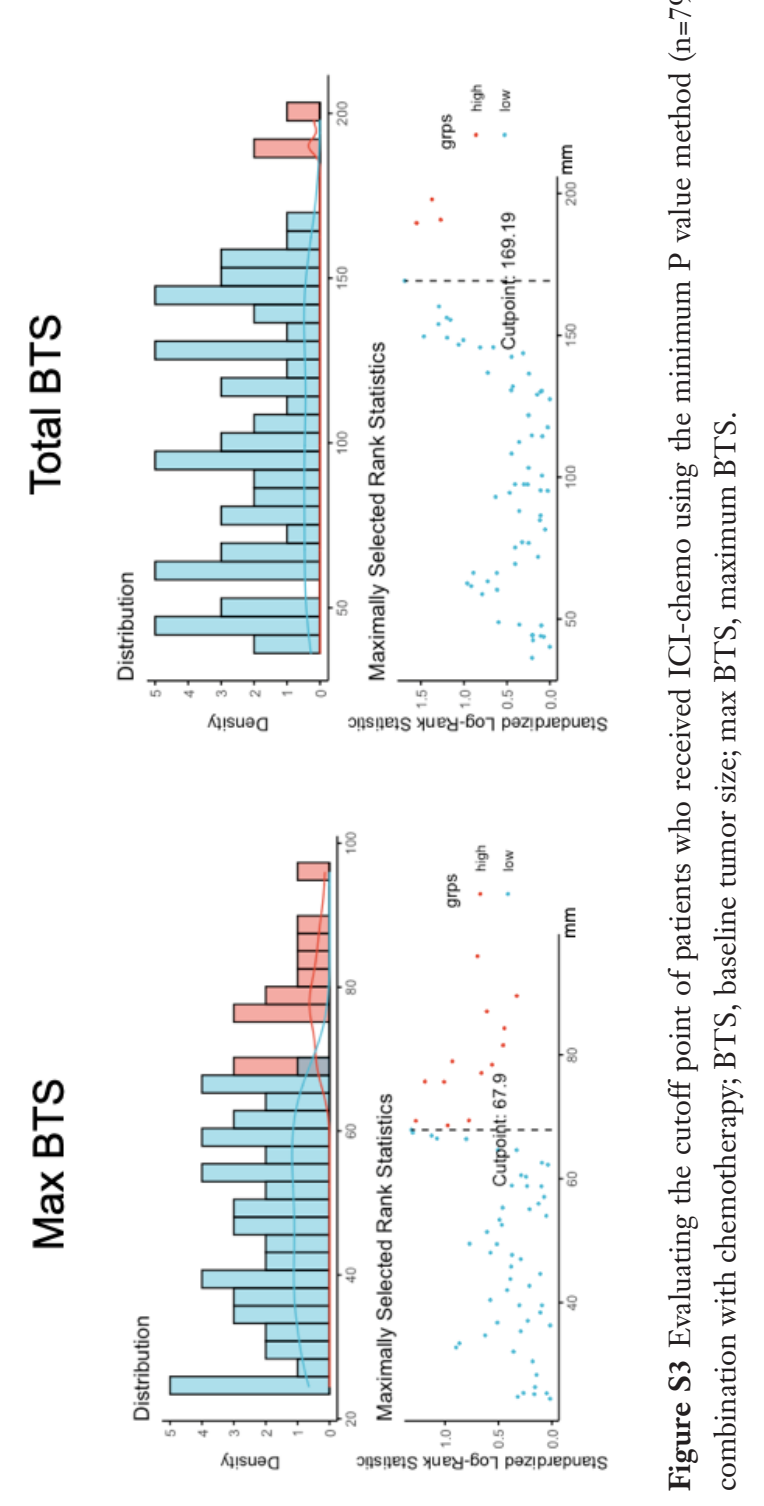
Table S3 Characteristics of patients who received ICI-chemo stratified by max BTS ( $n=79)$

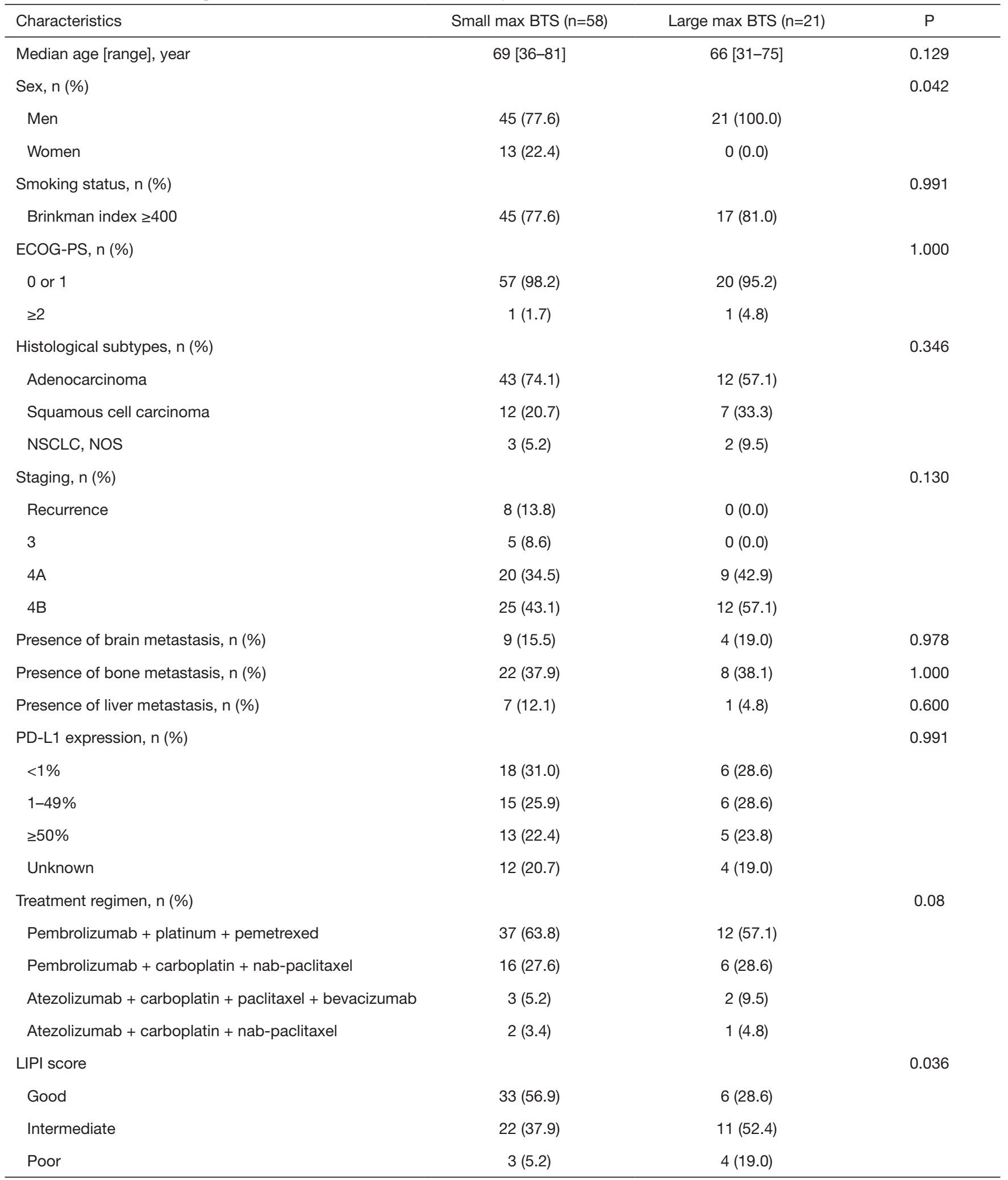

$\mathrm{ICl}$, immune checkpoint inhibitor; ICl-chemo, ICI in combination with chemotherapy; BTS, baseline tumor size; max BTS, maximum BTS; ECOG-PS, Eastern Cooperative Oncology Group performance status; NSCLC, non-small cell lung cancer; NOS, not otherwise specified; PD-L1, programmed death ligand-1; LIPI, lung immune prognostic index; IQR, interquartile range. 


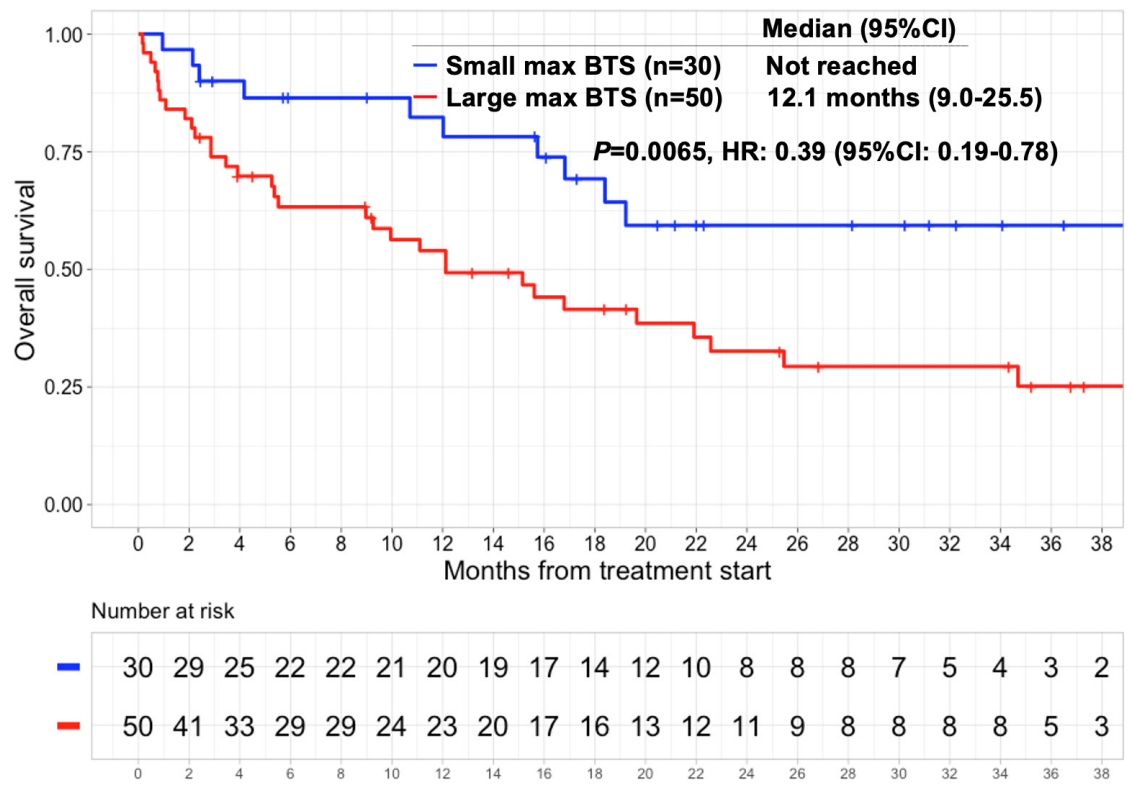

Figure S4 Kaplan-Meier estimates of the OS of patients who received ICI-mono $(\mathrm{n}=80)$. Comparison between the small and large max BTS groups. OS, overall survival; ICI, immune checkpoint inhibitor; ICI-mono, ICI monotherapy; BTS, baseline tumor size; max BTS, maximum BTS; HR, hazard ratio; CI, confidence interval.

Table S4 Best overall response to ICI-mono stratified by max BTS ( $\mathrm{n}=80)$

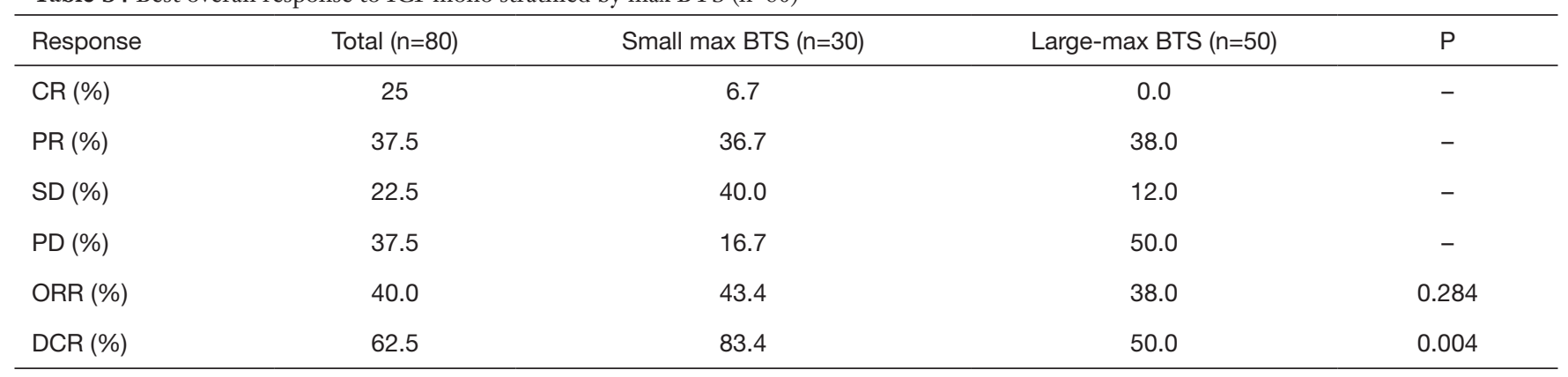

$\mathrm{ICI}$, immune checkpoint inhibitor; ICl-mono, ICI monotherapy; BTS, baseline tumor size; max BTS, maximum BTS; CR, complete response; PR, partial response; SD, stable disease; PD, progressive disease; ORR, overall response rate; DCR, disease control rate. 


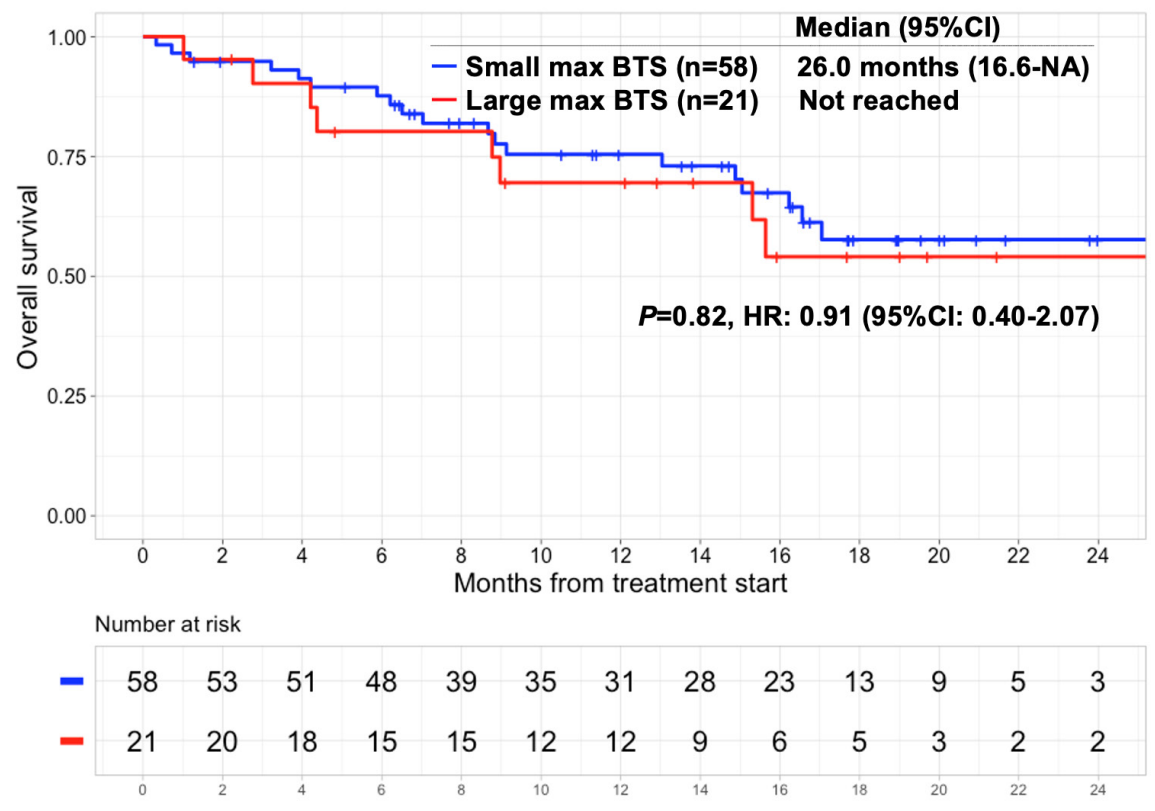

Figure S5 Kaplan-Meier estimates of the OS of patients who received ICI-chemo $(\mathrm{n}=79)$. Comparison between the small and large max BTS groups. OS, overall survival; ICI, immune checkpoint inhibitor; ICI-chemo, ICI in combination with chemotherapy; BTS, baseline tumor size; max BTS, maximum BTS; HR, hazard ratio; CI, confidence interval.

Table S5 Best overall response to ICI-chemo stratified by max BTS ( $\mathrm{n}=79)$

\begin{tabular}{lcccc}
\hline Response & Total $(n=79)$ & Small max BTS $(n=58)$ & Large max BTS $(n=21)$ & 0.0 \\
\hline CR (\%) & 0.0 & 0.0 & 76.2 & - \\
PR (\%) & 62.0 & 56.9 & 14.3 & - \\
SD (\%) & 22.8 & 25.9 & 9.5 & - \\
PD (\%) & 15.2 & 17.2 & 76.2 & 0.189 \\
ORR (\%) & 62.0 & 56.9 & 90.5 & 0.494 \\
DCR (\%) & 84.8 & 82.8 & & - \\
\hline
\end{tabular}

$\mathrm{ICl}$, immune checkpoint inhibitor; ICl-chemo, ICI in combination with chemotherapy; BTS, baseline tumor size; max BTS, maximum BTS; $\mathrm{CR}$, complete response; PR, partial response; SD, stable disease; PD, progressive disease; ORR, overall response rate; DCR, disease control rate. 
RMST: $\mathrm{t}=12$ months $\mathrm{ICl}+$ Chemo

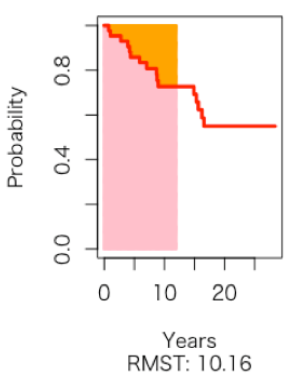

$\mathrm{ICl}$ alone

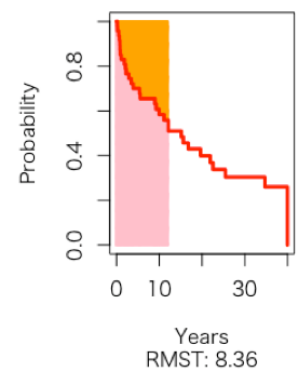

RMST between the 2 groups: 1.80 (95\% Cl 0.11-3.50)

$$
P=0.037
$$

RMST: $\mathrm{t}=18$ months $\mathrm{ICl}+$ Chemo
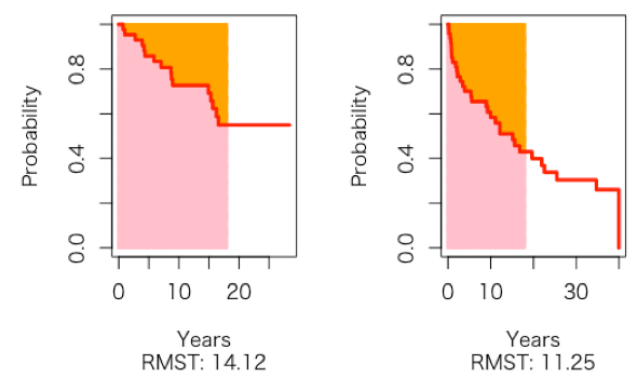

RMST between the 2 groups: 2.87 (95\%Cl 0.15-5.60)

$$
P=0.039
$$

Figure S6 RMST estimates of the OS of patients with max BTS $\geq 50 \mathrm{~mm}$ ( $\mathrm{n}=90)$. RMST, restricted mean survival time; ICI, immune checkpoint inhibitor; BTS, baseline tumor size; max BTS, maximum BTS; CI, confidence interval.

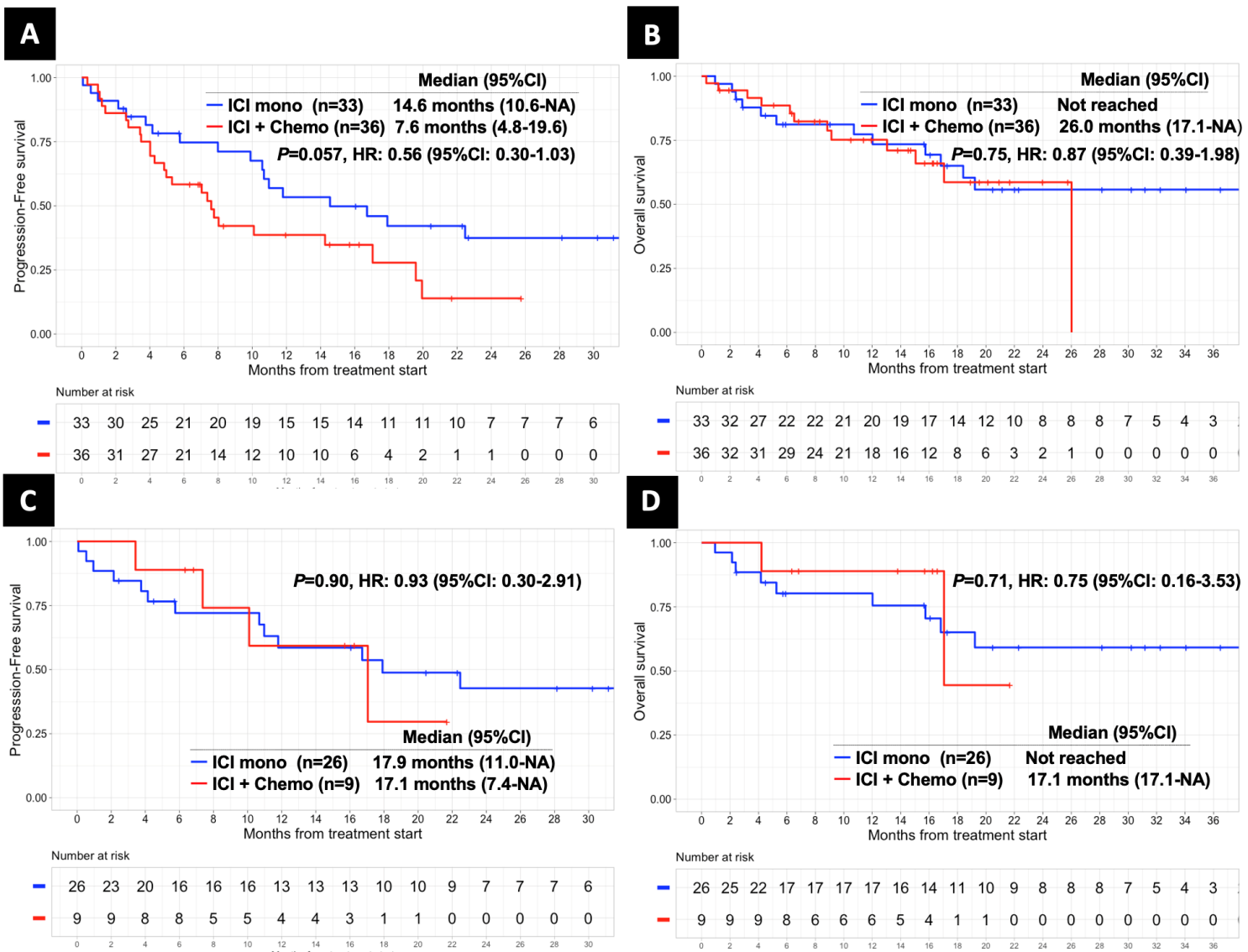

Figure S7 Comparison of the Kaplan-Meier estimates of patients with max BTS $<50 \mathrm{~mm}$ who received ICI-mono and ICI-chemo. (A) PFS $(\mathrm{n}=69)$. (B) OS (n=69). (C) PFS with PD-L1 score $\geq 50 \%$ group ( $\mathrm{n}=35)$. (D) OS with PD-L1 score $\geq 50 \%$ group (n=35). BTS, baseline tumor size; max BTS, maximum BTS; ICI, immune checkpoint inhibitor; ICI-mono, ICI monotherapy; ICI-chemo, ICI in combination with chemotherapy; PD-L1, programmed death ligand-1; HR, hazard ratio; CI, confidence interval. 
Table S6 Univariate and multivariable analysis of the PFS of patients with max BTS $<50 \mathrm{~mm}(\mathrm{n}=69)$

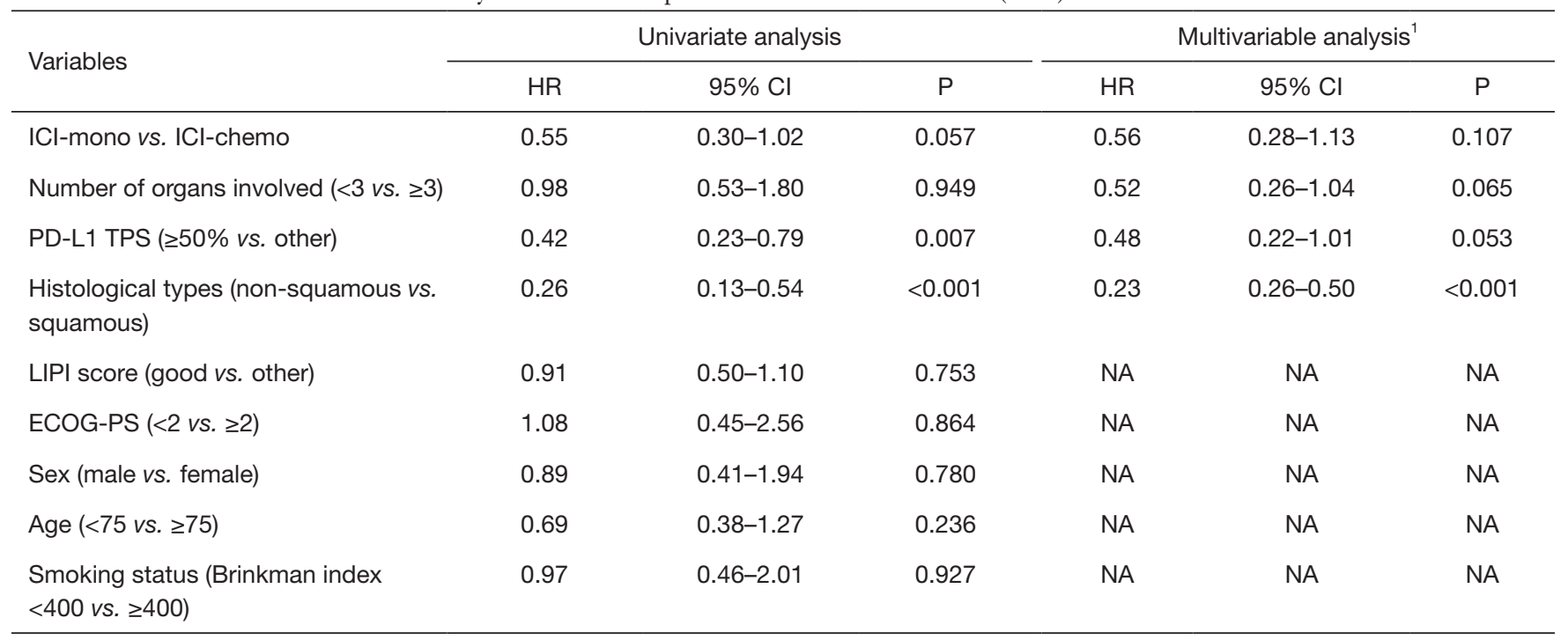

1, type of ICl treatment, PD-L1 TPS, variables with $\mathrm{P}<0.20$ in the univariate analysis were included. PFS, progression-free survival; BTS, baseline tumor size; max BTS, maximum BTS; ICI, immune checkpoint inhibitor; ICl-mono, ICl monotherapy; ICl-chemo, ICl in combination with chemotherapy; PD-L1, programmed death ligand-1; TPS, tumor proportion score; LIPI, lung immune prognostic index; ECOG-PS, Eastern Cooperative Oncology Group performance status; HR, hazard ratio; CI, confidence interval; NA, not available.

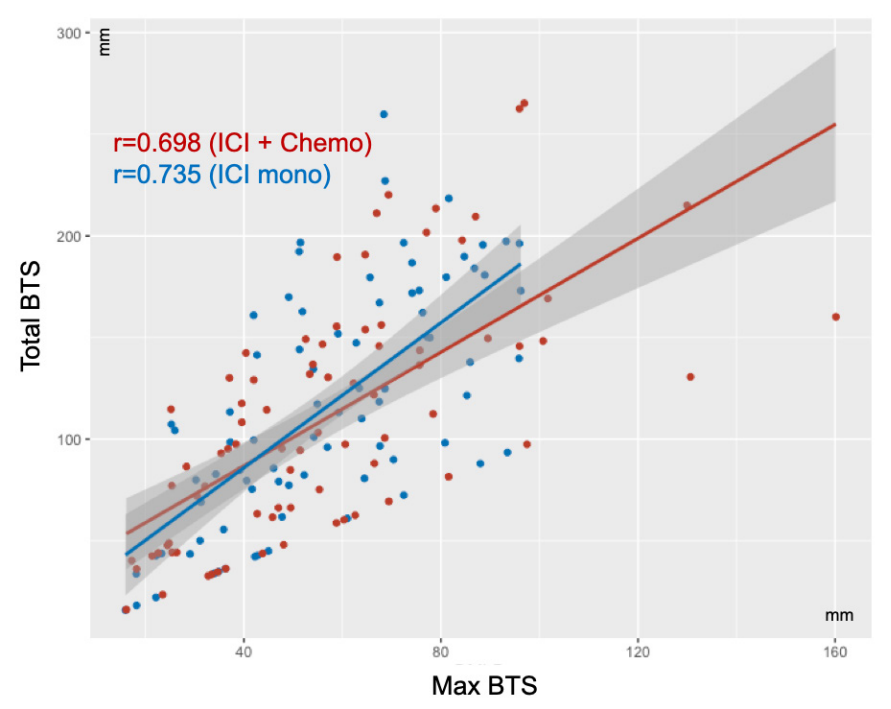

Figure S8 Correlative analysis of the max BTS and total BTS. The Spearman correlation coefficient (r) for the max BTS and total BTS in the ICI-mono group was $0.735(\mathrm{P}<0.0001)$. The Spearman correlation coefficient $(r)$ for the max BTS and total BTS in the ICI-chemo group was $0.698(\mathrm{P}<0.0001)$. BTS, baseline tumor size; max BTS, maximum BTS; ICI, immune checkpoint inhibitor; ICI-mono, ICI monotherapy; ICI-chemo, ICI in combination with chemotherapy. 